\title{
T-dualities and Doubled Geometry of the Principal Chiral Model
}

\author{
Vincenzo E. Marotta, ${ }^{a}$ Franco Pezzella ${ }^{b}$ and Patrizia Vitale ${ }^{b, c}$ \\ ${ }^{a}$ Department of Mathematics, Heriot-Watt University, \\ Colin Maclaurin Building, Riccarton, Edinburgh EH14 4AS, U.K. \\ ${ }^{b}$ INFN-Sezione di Napoli, \\ Complesso Universitario di Monte S. Angelo Edificio 6, via Cintia, 80126 Napoli, Italy \\ ${ }^{c}$ Dipartimento di Fisica "E. Pancini", Università di Napoli Federico II, \\ Complesso Universitario di Monte S. Angelo Edificio 6, via Cintia, 80126 Napoli, Italy \\ E-mail: vm34@hw.ac.uk, franco.pezzella@na.infn.it, \\ patrizia.vitale@na.infn.it
}

ABSTRACT: The Principal Chiral Model (PCM) defined on the group manifold of SU(2) is here investigated with the aim of getting a further deepening of its relation with Generalized Geometry and Doubled Geometry. A one-parameter family of equivalent Hamiltonian descriptions is analysed, and cast into the form of Born geometries. Then $O(3,3)$ duality transformations of the target phase space are performed and we show that the resulting dual models are defined on the group $\mathrm{SB}(2, \mathbb{C})$ which is the Poisson-Lie dual of $\mathrm{SU}(2)$ in the Iwasawa decomposition of the Drinfel'd double $\mathrm{SL}(2, \mathbb{C})$. A parent action with doubled degrees of freedom and configuration space $\operatorname{SL}(2, \mathbb{C})$ is then defined that reduces to either one of the dually related models, once suitable constraints are implemented.

Keywords: Sigma Models, Differential and Algebraic Geometry, String Duality

ARXIV EPRINT: 1903.01243 


\section{Contents}

1 Introduction 1

2 The Isotropic Rigid Rotator $\quad 6$

2.1 The dual model 9

$\begin{array}{lll}2.2 & \text { The generalized action } & 11\end{array}$

3 The Principal Chiral Model 14

3.1 The Hamiltonian formulation 16

$\begin{array}{lll}3.1 .1 & \text { Poisson-Lie structure } & 19\end{array}$

3.1.2 A family of Born geometries 20

4 Poisson-Lie dual models 23

4.1 The Lagrangian approach 26

4.1.1 The Hamiltonian description $\quad 27$

4.1.2 Dual Born geometry 28

5 Double principal chiral model $\quad 29$

$\begin{array}{ll}5.1 \text { The Lagrangian formalism } & 29\end{array}$

5.2 The Hamiltonian formalism 31

5.3 Recovering the Chiral Model on TSU(2) 31

6 Conclusions and outlook 33

A Poisson brackets 34

\section{Introduction}

Duality symmetries play a fundamental role in String Theory since they provide a powerful tool for investigating the structure of the target spacetime from the string point of view by relating, in the usual sigma-model approach, backgrounds which otherwise would be considered different. The Abelian T-duality [1-3] (where T stands for Target-space) is a well-known example of them. It is a distinctive symmetry of strings since, differently from particles, one-dimensional objects can wrap $d$ non-contractible cycles. This implies the presence of winding modes $w^{a}(a=1, \ldots, d)$ that have to be added to the ordinary momentum modes $p_{a}$ which take integer values along compact dimensions. On a $d$-torus $T^{d}$, the Abelian T-duality is an $O(d, d ; \mathbb{Z})$ string symmetry under, roughly speaking, the mapping of the radii of the compact dimensions into their inverse, together with the exchange of momentum and winding modes: in this way it establishes a connection between two apparently different but dual target spacetimes. From the sigma model point of view, the necessary condition to work out a dual to some background was, initially, that the latter possess an Abelian group of isometries [4-6] excluding in this way many physically relevant classical string vacua from being considered. 
After the work in ref. [7], it was understood that T-duality symmetries could also be associated with the non-Abelian isometries of the target manifold and, subsequently, the notion of Abelian and non-Abelian T-duality was extended to the one of Poisson-Lie T-duality [8-10]. Briefly, the term Abelian T-duality refers to the presence of global Abelian isometries in the target spaces of both the paired sigma models; non-Abelian T-duality refers to the existence of a global Abelian isometry on the target space of one of the two sigma-models and of a global non-Abelian isometry on the other. Finally, the Poisson Lie T-duality generalizes the previous definitions to all the other cases, including the one of a pair of sigma models both having non-Abelian isometries in their target spaces.

Beyond the string world-sheet action, a category of models that reveal themselves to be very helpful in understanding the above mentioned T-dualities is provided by sigma models whose target configuration space is a Lie group $G$ with $\mathfrak{g}$ its Lie algebra. These are the so-called Principal Chiral Models (PCM). Studying these models has led to abandoning the requirement of the existence of isometries for the target space as the condition for the existence of dual counterparts. Indeed, the relevant structure in this case reveals to be the one of Drinfel'd double for $G$ together with the well-established notion of Poisson-Lie symmetries [11-14]. The Drinfel'd double of a Lie group $G$ is defined as a Lie group $D$, with dimension twice the one of $G$, such that its Lie algebra $\mathfrak{d}$ can be decomposed into a pair of maximally isotropic sub-algebras, $\mathfrak{g}, \tilde{\mathfrak{g}}$ with respect to a non-degenerate invariant bilinear form on $\mathfrak{d}$, with $\mathfrak{g}, \tilde{\mathfrak{g}}$, respectively the Lie algebra of $G$ and its dual algebra. ${ }^{1}$ The dual algebra is endowed with a Lie bracket which has to be compatible with existing structures, in a precise sense which will be clarified below. Any such triple, $(\mathfrak{d}, \mathfrak{g}, \tilde{\mathfrak{g}})$, is referred to as a Manin triple. By exponentiation of $\tilde{\mathfrak{g}}$ one gets the dual Lie group $\tilde{G}$ such that locally $D \simeq G \times \tilde{G}$. The simplest example is the cotangent bundle of any $d$ dimensional Lie group $G, T^{*} G \simeq G \ltimes \mathbb{R}^{d}$, which we shall call the classical double, with trivial Lie bracket for the dual algebra $\tilde{\mathfrak{g}} \simeq \mathbb{R}^{d}$. For every decomposition of the Drinfel'd double $D$ into dually related subgroups $G, \tilde{G}$, it is possible to define a couple of PCM's having as target configuration space either of the two subgroups. Hence, every PCM has its dual counterpart for which the role of $G$ and its dual $\tilde{G}$ is interchanged. The set of all decompositions of $\mathfrak{d}$ into maximally isotropic subspaces (not necessarily subalgebras), plays the role of the modular space of sigma models mutually connected by an $O(d, d)$ transformation. In particular, for the manifest Abelian T-duality of the string model on the $d$-torus, the Drinfel'd double is $D=\mathrm{U}(1)^{2 d}$ and its modular space, is in one-to-one correspondence with $O(d, d ; \mathbb{Z})[10]$.

In this paper, we are going to show that the target phase space of the SU(2) PCM can actually be replaced by the Drinfel'd double of $\mathrm{SU}(2)$, namely the group $\mathrm{SL}(2, \mathbb{C})$, without modifying the dynamics. This observation, based on previous work by Rajeev $[15,16]$, is the main motivation for our interest in the model, since it allows to discuss Poisson-Lie T-duality, a generalization of Abelian T-duality, in a situation where it is a true symmetry of the undeformed dynamics. Later on we shall discuss more in detail this important point.

\footnotetext{
${ }^{1}$ An isotropic subspace of the Lie algebra $\mathfrak{d}$ is such that the bilinear form evaluated on any couple of vectors lying in that subspace vanishes; maximally isotropic means that the subspace cannot be enlarged while preserving the property of isotropy.
} 
Non-linear sigma models have been investigated in relation to Poisson-Lie duality, with or without reference to string theory, by many authors (see for example [17-27] and ref.s therein). A closer approach to the one which will be pursued in the paper has been adopted in $[28,29]$.

Another motivation for analyzing sigma models having as target phase-space a Drinfel'd double Lie group $D$, consists in the fact that it allows to establish some connections with Generalized Geometry (GG), by virtue of the fact that tangent and cotangent vector fields of the group manifold $G$ may be respectively related to the span of the Lie algebra $\mathfrak{g}$ and its dual, $\tilde{\mathfrak{g}}$. (Let us briefly recall that GG [30-32] contains, roughly speaking, two main ingredients - the first consists in replacing the tangent bundle $T$ of a manifold $M$ with $T \oplus T^{*}$, a bundle with the same base space $M$ but fibers given by the direct sum of tangent and cotangent spaces, and the second in replacing the Lie bracket among sections of $T$, that is vector fields, with the Courant bracket which involves vector fields and one-forms.) Moreover, Doubled Geometry (DG) may play a role in describing the generalized dynamics on the tangent bundle $T D \simeq D \times \mathfrak{d}$, which we shall do in order to describe within a single action both dually related models. Both GG and DG have revealed to be very suitable in describing the geometry of Double Field Theory (DFT) [33-37]. DFT provides a proposal to incorporate the Abelian T-duality of a compactified string on a $d$-torus $T^{d}$ in a $(\mathcal{G}, B)$ background as a manifest symmetry of the string effective field theory. More precisely, DFT is supposed to be an $O(d, d ; \mathbb{Z})$ manifest spacetime effective field theory that should derive from a manifestly T-dual invariant formulation of a string world-sheet action in which T-duality is made manifest. Such a formulation was proposed in ref.s $[3,38,39]$ and later developed in ref.s [40-47] (see more recent works in [48-53]). This string action has to contain information about windings and therefore it is based on two sets of coordinates: the usual string coordinates $x^{a}(\sigma, \tau)$ in the target space, having the momenta $p_{a}$ as conjugate, and the dual coordinates, $\tilde{x}_{a}(\sigma, \tau)$ having the winding modes as conjugate momenta. In this way the $O(d, d ; \mathbb{Z})$ duality results to be a manifest symmetry of the world-sheet action even paying the price of loosing the manifest covariance in the world-sheet two dimensions. A doubling of all the $N$ spacetime degrees of freedom in the low-energy effective action first occurred in ref.s [54-57] where a manifestly $O(N, N ; \mathbb{R})$ effective action in the target space was obtained, and such symmetry was realized linearly, loosing this time the manifest Lorentz invariance in the target space. In order to understand the role of Doubled and Generalized Geometry in a simpler context, the doubling of degrees of freedom has been analyzed in the context of finite-dimensional dynamical systems, such as the dynamics of a charged particle in presence of a uniform distribution of magnetic monopoles, in ref.s [5860], where the doubling is justified by the otherwise violated Jacobi identity for the algebra of observables. ${ }^{2}$ Moreover, it is worth mentioning that the occurrence of auxiliary degrees of freedom is also typical of other geometric theories, such as those based on Noncommutative Geometry. Noncommutative gauge theories require that the gauge group be enlarged (see for example [66] for a review). The differential calculus itself may be bigger than

\footnotetext{
${ }^{2}$ Violation of Jacobi identity can be related to the violation of associativity of the star product of the quantized theory [61]. See refs. [62-65] in relation to the problem of finding an associative star product for the electron-monopole system and related problems.
} 
in the commutative case (see $[67,68]$ for an example in three dimensions and [69] for an application to two-dimensional gauge theory). Renormalizability of noncommutative field theories entails the introduction of auxiliary parameters, such as for the Grosse-Wulkenhaar model [70], or the translation-invariant model [71, 72]. Last but not least, noncommutative extensions of Palatini-Holst theory of gravity imply the doubling of the tetrad degrees of freedom, leading to a bi-tetrad theory of gravity, with the manifestation of new duality symmetries $[73,74]$.

It should be clear that models whose carrier space of the dynamics is the manifold of a Lie group can be very helpful in better understanding T-duality and doubling of the degrees of freedom. The latter are naturally described in the framework outlined above, by generalizing the dynamics originally defined on $G$ to a dynamics on the Drinfel'd double $D$, through the introduction of a natural parent action; T-duality is naturally provided by the exchange of the two partner groups $G$ and $\tilde{G}$. The formulation of Double Field Theory on group manifolds, including its relation with Poisson-Lie symmetries, has been studied in ref.s [75, 76]. For recent results see ref.s [77, 78].

This is the second of a series of two papers. In the first one [79], we have studied the three-dimensional isotropic rigid rotator (IRR) that provides the simplest one-dimensional sigma model having $\mathbb{R}$ as a source space and the group manifold $\mathrm{SU}(2)$ as target configuration space. We have then introduced a model with target space the dual group $\operatorname{SB}(2, \mathbb{C})$ and considered the symmetry properties of the two models within an extended model on the Drinfel'd double $\mathrm{SL}(2, \mathbb{C})$, formulated in terms of a parent action. In particular, we have emphasized how a natural para-Hermitian structure emerges on the Drinfel'd double and can be used to provide a doubled formalism for the pair of theories. The IRR model is too simple to exhibit symmetry under duality transformation, being a $0+1$ field theory but it has paved the way for a genuine $1+1$ field theory, the SU(2) Principal Chiral Model which, while being modeled on the IRR system, certainly exhibits interesting properties under duality transformations: therefore, the SU(2) Principal Chiral Model is the topic of this second paper.

More precisely, we elaborate on an old intuition due to S.G. Rajeev which dates back to the 80 's $[15,16]$ where the principal $\mathrm{SU}(2)$ chiral model is shown to exhibit a whole one-parameter family of alternative Hamiltonians and alternative Poisson algebras, all equivalent from the point of view of the dynamics [also see ref. [80] where the construction is extended to the Wess-Zumino Witten model, and ref. [81] where the integrability is analyzed in terms of Lax pairs]. The model is described in the Hamiltonian approach by a pair of fields $J^{i}(t, \sigma), I_{i}(t, \sigma)$, the so-called currents, which are valued in the phase space $\mathrm{T}^{*} \mathrm{SU}(2)$, that we shall refer to as the target phase space. Let us briefly recall that, topologically, $\mathrm{T}^{*} \mathrm{SU}(2)$ is the manifold $S^{3} \times \mathbb{R}^{3}$, while, as a group, it is the semidirect product of $\mathrm{SU}(2)$ with the Abelian group $\mathbb{R}^{3}$. As a Poisson manifold it is known to be symplectomorphic to the group $\mathrm{SL}(2, C),{ }^{3}$ which should come with no surprise since the two have the same topology. Last but not least, $\mathrm{T}^{*} \mathrm{SU}(2)$ and $\mathrm{SL}(2, \mathbb{C})$ are both Drinfel'd doubles of the group $\mathrm{SU}(2)$ [11-14]. The former, which we shall call classical double, is the trivial one, with Abelian algebra of momenta and can be obtained from the latter via group contraction.

\footnotetext{
${ }^{3}$ when endowed with appropriate Poisson brackets [82].
} 
The many different geometric structures which are compatible with the manifold $S^{3} \times$ $\mathbb{R}^{3}$ will play a crucial role all over the paper. To start with, the whole construction relies on the generalization of the affine algebra of currents, associated with the semi-direct sum $\mathfrak{s u}(2)(\mathbb{R}) \ltimes \mathfrak{a}(\mathbb{R})$, being $\mathfrak{a}(\mathbb{R})$ an Abelian Lie algebra, to a fully semi-simple Kac-Moody algebra which is either $\mathfrak{s u}(2)(\mathbb{R}) \oplus \mathfrak{s u}(2)(\mathbb{R})$ or $\mathfrak{s l}(2, \mathbb{C})(\mathbb{R})$. Here by $\mathfrak{g}(\mathbb{R})$ we shall indicate the affine algebra associated to the Lie algebra $\mathfrak{g}$. Interestingly, this construction can be understood in terms of Born Geometry [83-87], which we shortly review and adapt to our model. By slightly generalizing the Poisson Kac-Moody algebra with the introduction of a second parameter, and performing an $O(3,3)$ transformation over the target phase space, we show that a family of sigma models with target configuration space the group manifold of $\mathrm{SB}(2, \mathbb{C})$ is obtained, which deserves the name of T-dual models. Moreover, the vanishing value of one of the two parameters corresponds to the original SU(2) PCM with canonical splitting of its current algebra, whereas the vanishing of the remaining parameter correctly reproduces the dual current algebra $\mathfrak{s} \mathfrak{b}(2, C)(\mathbb{R}) \ltimes \mathfrak{a}(\mathbb{R})$, but the Hamiltonian exhibits a singular behaviour which is yet to be understood.

Let us stress here that the one-parameter family of Hamiltonian models, re-proposed in eqs. (3.19)-(3.22), but already contained in [15, 16, 80], yields an equivalent description of the standard dynamics of the PCM. Namely, for each value of $\tau$ the dynamics is one and the same, up to rescaling the fields by appropriate factors of $\tau$. In this sense, it is different from the deformations introduced in ref. [88], which are true deformations of the dynamics. We prove explicitly that the same result holds for the two-parameter generalization represented by the algebra (4.1)-(4.3), upon rescaling and linear transforming $(I, K) \rightarrow(I, J)$.

The paper is organized as follows.

In section 2 the results obtained in ref. [79] are reviewed for the isotropic rigid rotator thought of as a dynamical model over the group manifold $\mathrm{SU}(2)$ with a dual partner defined on the dual group $\mathrm{SB}(2, \mathbb{C})$. The two groups appear in the Iwasawa decomposition of the Drinfel'd double $\operatorname{SL}(2, \mathbb{C})$ whose structure is recalled together with the one of its Lie algebra $\mathfrak{s l}(2, \mathbb{C}) \simeq \mathfrak{s u}(2) \bowtie \mathfrak{s} \mathfrak{b}(2, \mathbb{C})$.

In section 3 the generalization of the dynamics of the rigid rotor to the SU(2) Principal Chiral Model is described in the Lagrangian and Hamiltonian approach, with the introduction of the Poisson algebra of currents, which is the affine algebra $\mathfrak{s u}(2)(\mathbb{R}) \ltimes \mathfrak{a}(\mathbb{R})$. The existence of a whole one-parameter family of alternative Hamiltonians with a fully semi-simple affine algebra $\mathfrak{s l}(2, \mathbb{C})(\mathbb{R})$ is discussed and its interpretation in terms of Born geometries is analyzed.

In section 4 a family of T-dual models is introduced in the Hamiltonian formalism and it is shown that the target configuration space for the latter is the group manifold $\mathrm{SB}(2, \mathbb{C})$. In subsection 4.1 a different perspective is adopted. Analogously to what has been done for the Isotropic Rigid Rotator, a natural Lagrangian model is constructed directly on the dual group $\mathrm{SB}(2, \mathbb{C})$ and its relation to the dual models introduced previously is analyzed.

Finally, in the spirit of Double Field Theory, in order to build a model where the symmetries exhibited by the dynamics are manifest, a parent action is constructed in section 5 having as target configuration space the Drinfel'd double $\operatorname{SL}(2, \mathbb{C})$, hence doubling 
the degrees of freedom. From it, either of the dual partner models can be recovered, by gauging one of its global symmetries.

Conclusions and Outlook are reported in the final section 6. An appendix follows where the current algebras for all models considered are explicitly derived.

\section{The Isotropic Rigid Rotator}

In this section we shortly review the results obtained in ref. [79] for the isotropic rigid rotator as a dynamical model over the group manifold of SU(2), and its dual model having as configuration space the group manifold of the Lie-Poisson dual of $\mathrm{SU}(2)$, the group $\mathrm{SB}(2, \mathbb{C})$. Moreover, we briefly recall the Drinfel'd double structure of the group $\mathrm{SL}(2, \mathbb{C})$ and the bialgebra nature of its Lie algebra $\mathfrak{s l}(2, \mathbb{C}) \simeq \mathfrak{s u}(2) \bowtie \mathfrak{s} \mathfrak{b}(2, \mathbb{C})$.

The classical action which describes the dynamics can be chosen to be:

$$
S_{0}=-\frac{1}{4} \int_{\mathbb{R}} \operatorname{Tr}\left(\phi^{*}\left[g^{-1} \mathrm{~d} g\right] \wedge \underset{H}{*} \phi^{*}\left[g^{-1} \mathrm{~d} g\right]\right)=-\frac{1}{4} \int_{\mathbb{R}} \operatorname{Tr}\left(g^{-1} \frac{d g}{d t}\right)^{2} d t
$$

with $\phi: t \in \mathbb{R} \rightarrow g \in \mathrm{SU}(2),{ }_{H}^{*}$ the Hodge star operator on the source space $\mathbb{R},{ }_{H}^{*} d t=1$, Tr the trace over the Lie algebra and $g^{-1} d g$ the Maurer-Cartan left-invariant one-form on the group manifold. With an abuse of notation, the pull-back map $\phi^{*}$ will be omitted since now on.

Therefore the model can be regarded as a $(0+1)$-dimensional, group valued, field theory.

In order to motivate the interest for such a model, it is worth anticipating here that, with $g: \mathbb{R}^{1,1} \rightarrow \mathrm{SU}(2)$ and $\mathbb{R}^{1,1}$ the Minkowski spacetime, the action (2.1) generalizes to the one describing the Principal Chiral Model, that is to say, a non-linear sigma model with target space the group manifold of $\mathrm{SU}(2)$.

By choosing the parametrization $g=y^{0} \sigma_{0}+i y^{i} \sigma_{i}$, with $\left(y^{0}\right)^{2}+\sum_{i}\left(y^{i}\right)^{2}=1$ and $\sigma_{0}$ the identity matrix, $\sigma_{i}$ Pauli matrices and the inverse relations

$$
y^{i}=-\frac{i}{2} \operatorname{Tr} g \sigma_{i}, \quad y^{0}=\frac{1}{2} \operatorname{Tr} g \sigma_{0}, \quad i=1, \ldots, 3,
$$

one has:

$$
g^{-1} \dot{g}=i\left(y^{0} \dot{y}^{i}-y^{i} \dot{y}^{0}+\epsilon_{j k}{ }^{i} y^{j} \dot{y}^{k}\right) \sigma_{i}:=i \dot{Q}^{i} \sigma_{i}
$$

being

$$
\dot{Q}^{i}=y^{0} \dot{y}^{i}-y^{i} \dot{y}^{0}+\epsilon_{j k}^{i} y^{j} \dot{y}^{k}
$$

the left generalized velocities. ${ }^{4}$ The Lagrangian then reads as:

$$
\mathcal{L}_{0}=\frac{1}{2}\left(y^{0} \dot{y}^{j}-y^{j} \dot{y}^{0}+\epsilon_{k l}{ }^{j} y^{k} \dot{y}^{l}\right)\left(y^{0} \dot{y}^{r}-y^{r} \dot{y}^{0}+\epsilon_{p q}{ }^{r} y^{p} \dot{y}^{q}\right) \delta_{i r}:=\frac{1}{2} \dot{Q}^{j} \dot{Q}^{r} \delta_{j r} .
$$

This yields the following equations of motion:

$$
\mathrm{L}_{\Gamma} \dot{Q}^{i}=0, \quad \text { or equivalently } \mathrm{L}_{\Gamma}\left(g^{-1} \frac{d g}{d t}\right)=0
$$

with $\mathrm{L}_{\Gamma}$ the Lie derivative with respect to $\Gamma=\frac{d}{d t}$.

\footnotetext{
${ }^{4}$ Had we chosen to work with the right-invariant Maurer-Cartan one-form we would have introduced right generalized velocities.
} 
The cotangent bundle (left) coordinates are represented by $\left(Q^{i}, I_{i}\right)$ with $I_{i}$ being the left conjugate momenta:

$$
I_{i}=\frac{\partial \mathcal{L}_{0}}{\partial \dot{Q}^{i}}=\delta_{i j} \dot{Q}^{j}
$$

The Hamiltonian is thus $\mathcal{H}_{0}=\frac{1}{2} I_{i} I_{j} \delta^{i j}$ with Poisson brackets (see ref. [79] for details) given by:

$$
\begin{aligned}
\left\{y^{i}, y^{j}\right\} & =0 \\
\left\{I_{i}, I_{j}\right\} & =\epsilon_{i j}{ }^{k} I_{k} \\
\left\{y^{i}, I_{j}\right\} & =\delta_{j}^{i} y^{0}+\epsilon_{j k}{ }^{i} y^{k} \quad \text { or } \quad\left\{g, I_{j}\right\}=i g \sigma_{j} g
\end{aligned}
$$

which lead to the dynamics described by the following equations:

$$
\dot{I}_{i}=0, \quad g^{-1} \dot{g}=i I_{i} \delta^{i j} \sigma_{j} .
$$

The fiber coordinates $I_{i}$ are associated with the angular momentum components and the base space coordinates $g \equiv\left(y^{0}, y^{i}\right)$ to the orientation of the rotator.

As well-known, $I_{i}$ are constants of the motion, while $g$ undergoes a uniform precession. Remarks:

- As a group $\mathrm{T}^{*} \mathrm{SU}(2)$ is the semi-direct product $\mathrm{SU}(2) \ltimes \mathbb{R}^{3}$ with Lie algebra $\mathfrak{s u}(2) \ltimes \mathbb{R}^{3}$ and Lie brackets given by:

$$
\left[L_{i}, L_{j}\right]=\epsilon_{i j}{ }^{k} L_{k} \quad\left[T_{i}, T_{j}\right]=0 \quad\left[L_{i}, T_{j}\right]=\epsilon_{i j}{ }^{k} T_{k} .
$$

Here $L_{i}, T_{i}, i=1,2,3$ generate respectively the algebra $\mathfrak{s u}(2)$ and the algebra $\mathbb{R}^{3}$.

- The non-trivial Poisson brackets (2.7)-(2.9) are the Kirillov-Souriau-Konstant (KSK) brackets on the dual algebra $\tilde{\mathfrak{g}}$.

Starting from these remarks, in ref. [89] the carrier space of the dynamics has been generalized to $\mathrm{SL}(2, \mathbb{C})$, the non-trivial Drinfel'd double of $\mathrm{SU}(2)$, which, roughly speaking, can be obtained by deforming the Abelian subgroup $\mathbb{R}^{3}$ of the semi-direct product above, and a similar generalization has been proposed for the Principal Chiral Model $[15,16]$ and the Wess-Zumino-Witten Model [80].

The algebra $\mathfrak{s l}(2, \mathbb{C})$ is usually described in terms of the generators $e_{i}=\sigma_{i} / 2, b_{i}=i e_{i}$, $i=1,2,3$, with Lie brackets

$$
\left[e_{i}, e_{j}\right]=i \epsilon_{i j}{ }^{k} e_{k}, \quad\left[e_{i}, b_{j}\right]=i \epsilon_{i j}^{k} b_{k}, \quad\left[b_{i}, b_{j}\right]=-i \epsilon_{i j}{ }^{k} e_{k} .
$$

It is equipped with two non-degenerate invariant scalar products:

$$
\langle u, v\rangle=2 \operatorname{Im}(\operatorname{Tr}(u v)) \quad \forall u, v \in \mathfrak{s l}(2, \mathbb{C})
$$

and

$$
(u, v)=2 \operatorname{Re}(\operatorname{Tr}(u v)) \quad \forall u, v \in \mathfrak{s l}(2, \mathbb{C}) .
$$


With respect to the first one (the Cartan-Killing metric), one has two maximal isotropic subspaces, spanned by $\left\{e_{i}\right\}$, and the linear combination

$$
\tilde{e}^{i}=b_{i}-\epsilon_{i j 3} e_{j}
$$

Indeed the following relations hold:

$$
\left\langle e_{i}, e_{j}\right\rangle=\left\langle\tilde{e}^{i}, \tilde{e}^{j}\right\rangle=0 \quad \text { and } \quad\left\langle e_{i}, \tilde{e}^{j}\right\rangle=\delta_{i}^{j} .
$$

The generators $\left\{e_{i}\right\},\left\{\tilde{e}^{i}\right\}$ span two non-commuting subalgebras of $\mathfrak{s l}(2, \mathbb{C})$ with Lie brackets:

$$
\left[e_{i}, e_{j}\right]=i \epsilon_{i j}{ }^{k} e_{k}, \quad\left[\tilde{e}^{i}, e_{j}\right]=i \epsilon_{j k}{ }^{i} \tilde{e}^{k}+i e_{k} f_{j}^{k i}, \quad\left[\tilde{e}^{i}, \tilde{e}^{j}\right]=i f_{k}^{i j} \tilde{e}^{k} .
$$

In particular, $\left\{\tilde{e}^{i}\right\}$ span the Lie algebra of $\mathrm{SB}(2, \mathbb{C})$, the dual group of $\mathrm{SU}(2)$ with

$$
f_{k}^{i j}=\epsilon^{i j l} \epsilon_{l 3 k} .
$$

Each algebra acts on the other one non-trivially by coadjoint action, as it can be read from eq. (2.16) and therefore we denote the total algebra by $\mathfrak{s l}(2, \mathbb{C})=\mathfrak{s u}(2) \bowtie \mathfrak{s} \mathfrak{b}(2, \mathbb{C})$, with $\bowtie$ generalizing the semi-direct sum.

Summarizing:

- $\mathfrak{s l}(2, \mathbb{C})$ can be endowed with a Lie bialgebra structure;

- the role of $\mathfrak{s u}(2)$ and its dual algebra can be interchanged.

The triple $(\mathfrak{s l}(2, \mathbb{C}), \mathfrak{s u}(2), \mathfrak{s} \mathfrak{b}(2, \mathbb{C}))$ is called a Manin triple.

The construction can be generalized to any Lie group $G$. Given $\mathfrak{d}=\mathfrak{g} \bowtie \tilde{\mathfrak{g}}$, the group $D$ with Lie algebra $\mathfrak{d}$ is the Drinfel'd double and $G, \tilde{G}$ are dual groups. For $f^{i j}{ }_{k}=0 \quad D \rightarrow$ $T^{*} G$, while for $c_{i j}{ }^{k}=0 \quad D \rightarrow T^{*} \tilde{G}$, with $c_{i j}{ }^{k}$ the structure constants of $\mathfrak{g}$ and $f^{i j}{ }_{k}$ the structure constants of $\tilde{\mathfrak{g}}$. Therefore $D$ generalizes both the cotangent bundle of $G$ and of $\tilde{G}$.

The bialgebra structure induces Poisson structures on the group manifold of the double $D$ which generalize both those of $T^{*} G$ and of $T^{*} \tilde{G}$ and reproduce the KSK brackets on coadjoint orbits of $G, \tilde{G}$ when $f^{i j}{ }_{k}=0, c_{i j}{ }^{k}=0$ respectively. For $\gamma \in D$ and being $r=\lambda \tilde{e}^{i} \otimes e_{i}$ with $\lambda \in \mathbb{R}$ the classical Yang-Baxter matrix, the brackets

$$
\left\{\gamma_{1}, \gamma_{2}\right\}=-\gamma_{1} \gamma_{2} r^{*}-r \gamma_{1} \gamma_{2}
$$

where $\gamma_{1}=\gamma \otimes 1, \gamma_{2}=1 \otimes \gamma_{2}, r^{*}=-\lambda e_{i} \otimes \tilde{e}^{i}$, can be shown to define a Poisson structure on the group manifold [13, 90]. The group $D$ equipped with this Poisson bracket is also called the Heisenberg double of $G$.

By writing $\gamma$ as $\gamma=\tilde{g} g$, with $\tilde{g} \in \tilde{G}, g \in G$, it can be shown that these brackets are compatible with the following ones:

$$
\begin{aligned}
& \left\{\tilde{g}_{1}, \tilde{g}_{2}\right\}=-\left[r, \tilde{g}_{1} \tilde{g}_{2}\right] \\
& \left\{\tilde{g}_{1}, g_{2}\right\}=-\tilde{g}_{1} r g_{2} \quad\left\{g_{1}, \tilde{g}_{2}\right\}=-\tilde{g}_{2} r^{*} g_{1} \\
& \left\{g_{1}, g_{2}\right\}=\left[r^{*}, g_{1} g_{2}\right],
\end{aligned}
$$


where (2.19) and (2.21) are the Sklyanin brackets [91,92]. Let us now specify to the example at hand with $G=\mathrm{SU}(2)$ and $\tilde{G}=\mathrm{SB}(2, \mathbb{C})$. One can choose for the latter the parametrization $\tilde{g}=2\left(u_{0} e^{0}+i u_{i} \tilde{e}^{i}\right)$ with $u_{0}^{2}-u_{3}^{2}=1$ and $\tilde{e}^{0}=\mathbb{1} / 2, \tilde{e}^{i}$ being generators of the Lie algebra $\mathfrak{s} \mathfrak{b}(2, \mathbb{C})$, which is going to be specified below. By expanding $\tilde{g}$ as a function of the parameter $\lambda, \tilde{g}(\lambda)=1+i \lambda I_{i} e^{i}+\mathcal{O}\left(\lambda^{2}\right)$, while keeping $g=y^{0} \sigma_{0}+i y^{i} \sigma_{i}$, one obtains, in the limit $\lambda \rightarrow 0$ :

$$
\begin{aligned}
\left\{I_{i}, I_{j}\right\} & =\epsilon_{i j}^{k} I_{k} \\
\left\{I_{i}, y^{0}\right\} & =i y^{j} \delta_{i j} \quad\left\{I_{i}, y^{j}\right\}=i y^{0} \delta_{i}^{j}-\epsilon_{i k}^{j} y^{k} \\
\left\{y^{0}, y^{j}\right\} & =\left\{y^{i}, y^{j}\right\}=0+O(\lambda)
\end{aligned}
$$

which reproduce correctly the canonical Poisson brackets on the cotangent bundle of SU(2). Consider now $r^{*}$ as an independent solution of the Yang-Baxter equation $r^{*} \rightarrow \rho=\mu e_{k} \otimes e^{k}$ with $\mu \in \mathbb{R}$ and expand $g \in \mathrm{SU}(2)$ as a function of the parameter $\mu, g=\mathbf{1}+i \mu \tilde{I}^{i} e_{i}+O\left(\mu^{2}\right)$ while keeping $\tilde{g}$ in its original parametrization. By repeating the same analysis as above, one gets back the canonical Poisson structure on $\mathrm{T}^{*} \mathrm{SB}(2, C)$, with position coordinates and momenta now interchanged. In particular we note that:

$$
\left\{\tilde{I}^{i}, \tilde{I}^{j}\right\}=f^{i j}{ }_{k} \tilde{I}^{k} .
$$

Furthermore, it is possible to consider a different Poisson structure on the double [13], given by:

$$
\left\{\gamma_{1}, \gamma_{2}\right\}=\frac{\lambda}{2}\left[\gamma_{1}\left(r^{*}-r\right) \gamma_{2}-\gamma_{2}\left(r^{*}-r\right) \gamma_{1}\right]
$$

This is the one that correctly dualizes the bialgebra structure on $\mathfrak{d}$ when evaluated at the identity of the group $D$. Indeed, by expanding $\gamma \in D$ as $\gamma=\mathbf{1}+i \lambda I_{i} \tilde{e}^{i}+i \lambda \tilde{I}^{i} e_{i}$ and rescaling $r, r^{*}$ by the same parameter $\lambda$, one can show that:

$$
\begin{aligned}
& \left\{I_{i}, I_{j}\right\}=\epsilon_{i j}{ }^{k} I_{k} ; \quad\left\{\tilde{I}^{i}, \tilde{I}^{j}\right\}=f^{i j}{ }_{k} \tilde{I}^{k} \\
& \left\{I_{i}, \tilde{I}^{j}\right\}=-f^{j k}{ }_{i} I_{k}-\tilde{I}^{k} \epsilon_{k i}{ }^{j}
\end{aligned}
$$

which are the Poisson brackets induced by the Lie bi-algebra structure of the double. One can see that the fiber coordinates $I_{i}$ and $\tilde{I}^{j}$ play a symmetric role. Moreover, since the fiber coordinate $\tilde{I}^{i}$ appears in the expansion of $g$, it can also be thought of as the fiber coordinate of the tangent bundle TSU(2), so that the couple $\left(I_{i}, \tilde{I}^{i}\right)$ identifies the fiber coordinate of the generalized bundle $T \oplus T^{*}$ over $\mathrm{SU}(2)$.

\subsection{The dual model}

Let us now go back to the two scalar products in the Lie bialgebra, (2.12)-(2.13). With respect to the second scalar product, one has another splitting:

$$
\left(e_{i}, e_{j}\right)=-\left(b_{i}, b_{j}\right)=\delta_{i j}, \quad\left(e_{i}, b_{j}\right)=0
$$

with maximal isotropic subspaces: $f_{i}^{ \pm}=\frac{1}{\sqrt{2}}\left(e_{i} \pm b_{i}\right)$. The following doubled notation can be introduced:

$$
e_{I}=\left(\begin{array}{c}
e_{i} \\
\tilde{e}^{i}
\end{array}\right), \quad e_{i} \in \mathfrak{s u}(2), \quad \tilde{e}^{i} \in \mathfrak{s} \mathfrak{b}(2, \mathbb{C}) .
$$


The first scalar product then becomes:

$$
\left\langle e_{I}, e_{J}\right\rangle=\eta_{I J}=\left(\begin{array}{cc}
0 & \delta_{i}^{j} \\
\delta_{j}^{i} & 0
\end{array}\right)
$$

which is $O(3,3)$ invariant by construction.

The second scalar product yields:

$$
\left(e_{I}, e_{J}\right)=\left(\begin{array}{cc}
\delta_{i j} & \epsilon_{i p 3} \delta^{p j} \\
\delta^{i p} \epsilon_{j p 3} & \delta^{i j}-\epsilon^{i k 3} \delta_{k l} \epsilon^{j l 3}
\end{array}\right) .
$$

With $C_{+}, C_{-}$being the two subspaces spanned by $\left\{e_{i}\right\},\left\{b_{i}\right\}$ respectively, one can notice that the splitting $\mathfrak{d}=C_{+} \oplus C_{-}$defines a positive definite metric on $\mathfrak{d}$ via:

$$
\mathcal{H}=(,)_{C_{+}}-(,)_{C_{-}} .
$$

It is immediate to check that the metric $\mathcal{H}$, that will be indicated since now on by double round brackets:

$$
\left(\left(e_{i}, e_{j}\right)\right):=\left(e_{i}, e_{j}\right) ; \quad\left(\left(b_{i}, b_{j}\right)\right):=-\left(b_{i}, b_{j}\right) ; \quad\left(\left(e_{i}, b_{j}\right)\right):=\left(e_{i}, b_{j}\right)=0
$$

satisfies

$$
\mathcal{H}^{T} \eta \mathcal{H}=\eta
$$

namely $\mathcal{H}$ is a pseudo-orthogonal $O(3,3)$ metric. The sum $\alpha \eta+\beta \mathcal{H}$ is a non-degenerate metric for a suitable choice of the parameters $\alpha, \beta$. Notice that the latter can be rewritten as

$$
((u, v))=2 \operatorname{Re} \operatorname{Tr}\left[u^{\dagger} v\right]
$$

showing that it is in general not invariant.

In ref. [79] a dynamical model has been introduced on the cotangent bundle of the dual group $\mathrm{T}^{*} \mathrm{SB}(2, \mathbb{C})$, with action given by:

$$
\tilde{S}_{0}=-\frac{1}{4} \int_{\mathbb{R}} \mathcal{T} r\left[\phi^{*}\left(\tilde{g}^{-1} \mathrm{~d} \tilde{g}\right) \wedge \underset{H}{*} \phi^{*}\left(\tilde{g}^{-1} \mathrm{~d} \tilde{g}\right)\right]
$$

with $\phi: t \in \mathbb{R} \rightarrow \tilde{g} \in \mathrm{SB}(2, \mathbb{C}), \phi^{*}$ the pull-back map, $\tilde{g}=2\left(u_{0} \tilde{e}^{0}+i u_{i} \tilde{e}^{i}\right)$, and $u_{0}^{2}-u_{3}^{2}=1$. $\mathcal{T} r$ was chosen to be the non-degenerate product $(2.30) \mathcal{T} r:=(()$,$) , which is however only$ invariant under left $\mathrm{SB}(2, \mathbb{C})$ action [79]. The latter defines a non-degenerate left-invariant metric over the fibers,

$$
h^{i j}:=\delta^{i j}+\epsilon^{i k 3} \delta_{k l} \epsilon^{j l 3}
$$

so that the Lagrangian can be rewritten as:

$$
\tilde{L}_{0}=\frac{1}{2} \dot{\tilde{Q}}_{i} h^{i j} \dot{\tilde{Q}}_{j}
$$

with $\dot{\tilde{Q}}_{i}=u_{0} \dot{u}_{i}-u_{i} \dot{u}_{0}+f^{j k}{ }_{i} u_{j} \dot{u}_{k}$ being the left tangent bundle coordinates defined through the Maurer-Cartan form:

$$
\tilde{g}^{-1} \dot{\tilde{g}}=\dot{\tilde{Q}}_{i} \tilde{e}^{i}
$$


In analogy with the case of the rigid rotor, the equations of motion are easily retrieved:

$$
\mathrm{L}_{\Gamma} \dot{\tilde{Q}}_{j} h^{j i}-\dot{\tilde{Q}}_{p} \dot{\tilde{Q}}_{q} f_{k}^{i p} h^{q k}=0
$$

where $L_{\Gamma}$ is the Lie derivative with respect to $\Gamma=\frac{d}{d t}$.

Left momenta living on the cotangent bundle are introduced through a Legendre transform:

$$
\begin{aligned}
\tilde{I}^{i} & =\frac{\partial \tilde{\mathcal{L}}_{0}}{\partial \dot{\tilde{Q}}_{i}}=h^{i j} \dot{\tilde{Q}}_{j} \\
\tilde{H}_{0} & =\frac{1}{2} \tilde{I}^{i} h_{i j} \tilde{I}^{j}
\end{aligned}
$$

with

$$
h_{i j}=\left(\delta_{i j}-\frac{1}{2} \epsilon_{i k 3} \delta^{k l} \epsilon_{j l 3}\right)
$$

the inverse metric. By means of the Poisson brackets (see ref. [79] for details):

$$
\begin{aligned}
& \left\{u_{i}, u_{j}\right\}=0 \\
& \left\{\tilde{I}^{i}, \tilde{I}^{j}\right\}=f^{i j}{ }_{k} \tilde{I}^{k} \\
& \left\{u_{i}, \tilde{I}^{j}\right\}=\delta_{i}^{j} u_{0}-f^{j k}{ }_{i} u_{k}
\end{aligned}
$$

one obtains the Hamiltonian dynamics

$$
\dot{\tilde{I}}^{j}=f^{j k}{ }_{l}^{l} \tilde{I}^{r} h_{r k}
$$

expressing that the Hamiltonian is not invariant under right $\mathrm{SB}(2, \mathbb{C})$ action. By introducing right momenta we would get instead $\underline{\dot{I}}^{j}=0$, consistently with the invariance of the Hamiltonian under left action.

The Poisson brackets of both models, reported in eqs. (2.7)-(2.9) and (2.40)(2.42), have the structure of a semi-direct product. Moreover the Poisson brackets for the momenta can be retrieved by the Poisson-Lie bracket of the dual group (resp. eq. (2.19) for the Poisson bracket of the $\mathrm{SU}(2)$ momenta, eq. (2.21) for the Poisson bracket of the $\mathrm{SB}(2, \mathbb{C})$ momenta). It is therefore natural to describe this structure as a kind of Poisson-Lie duality and look for a generalized model over the group manifold of the double group, which encodes both models once suitably constrained.

\subsection{The generalized action}

In ref. [79] a generalized action with doubled degrees of freedom has been introduced in the form:

$$
\mathcal{S}=\int k_{1}\left\langle\gamma^{-1} \mathrm{~d} \gamma \wedge \underset{H}{*} \gamma^{-1} \mathrm{~d} \gamma\right\rangle+k_{2}\left(\left(\gamma^{-1} \mathrm{~d} \gamma \wedge \underset{H}{*} \gamma^{-1} \mathrm{~d} \gamma\right)\right)
$$

with $\gamma \in \mathrm{SL}(2, \mathbb{C}), e_{I}=\left(e_{i}, \tilde{e}^{i}\right), \gamma^{-1} \mathrm{~d} \gamma=\dot{\mathbf{Q}}^{I} e_{I} d t \equiv\left(A^{i} e_{i}+B_{i} \tilde{e}^{i}\right) d t$ the left-invariant Maurer-Cartan one-form on $\mathrm{SL}(2, \mathbb{C})$ pulled-back to $\mathbb{R}$ and $\left(A^{i}, B_{i}\right)$ fiber coordinates of $\operatorname{TSL}(2, \mathbb{C})$. They are obtained by means of the scalar product $(2.12)$ according to:

$$
A^{i}=2 \operatorname{Im} \operatorname{Tr}\left(\gamma^{-1} \dot{\gamma} \tilde{e}^{i}\right) ; \quad B_{i}=2 \operatorname{Im} \operatorname{Tr}\left(\gamma^{-1} \dot{\gamma} e_{i}\right)
$$


Upon introducing $k=k_{1} / k_{2}$, the Lagrangian can be rewritten in terms of the left generalized coordinates $\dot{\mathbf{Q}}^{I}$ as follows:

$$
L=\frac{1}{2}\left(k \eta_{I J}+\mathcal{H}_{I J}\right) \dot{\mathbf{Q}}^{I} \dot{\mathbf{Q}}^{J}
$$

with

$$
k \eta_{I J}+\mathcal{H}_{I J}=\left(\begin{array}{cc}
\delta_{i j} & \left(k \delta_{i p}+\epsilon_{i p 3}\right) \delta^{p j} \\
\delta^{i p}\left(k \delta_{p j}-\epsilon_{j p 3}\right) & \delta^{i j}+\epsilon^{i k 3} \delta_{k l} \epsilon^{j l 3}
\end{array}\right) .
$$

The equations of motion are:

$$
\mathrm{L}_{\Gamma} \dot{\mathbf{Q}}^{I}\left(k \eta_{I J}+\mathcal{H}_{I J}\right)-\dot{\mathbf{Q}}^{P} \dot{\mathbf{Q}}^{Q} C_{I P}{ }^{K}\left(k \eta_{Q K}+\mathcal{H}_{Q K}\right)=0
$$

where $C_{I P}{ }^{K}$ are the structure constants of $\mathfrak{s l}(2, \mathbb{C})$. The matrix $k \eta_{I J}+\mathcal{H}_{I J}$ is non-singular provided $k^{2} \neq 1$, which is going to be assumed from now on. In the doubled description introduced above, the left generalized momenta are represented by:

$$
\mathbf{P}_{I}=\frac{\partial L}{\partial \dot{\mathbf{Q}}^{I}}=\left(k \eta_{I J}+\mathcal{H}_{I J}\right) \dot{\mathbf{Q}}^{J}
$$

The Hamiltonian reads then as:

$$
\hat{H}=\left(\mathbf{P}_{I} \dot{\mathbf{Q}}^{I}-L\right)_{\mathbf{P}}=\frac{1}{2}\left[(k \eta+\mathcal{H})^{-1}\right]^{I J} \mathbf{P}_{I} \mathbf{P}_{J}
$$

with

$$
\left[(k \eta+\mathcal{H})^{-1}\right]^{I J}=\frac{1}{1-k^{2}}\left(\begin{array}{cc}
\delta^{i j}+\epsilon^{i l 3} \delta_{l k} \epsilon^{j k 3} & -\left(\epsilon^{i p 3}+k \delta^{i p}\right) \delta_{p j} \\
\left(\epsilon_{i p 3}-k \delta_{i p}\right) \delta^{p j} & \delta_{i j}
\end{array}\right) .
$$

In terms of the components $I_{i}, \tilde{I}^{j}$ of $\mathbf{P}_{I}$ the Hamiltonian can be rewritten as:

$$
\hat{H}=\frac{1}{2\left(1-k^{2}\right)}\left(\left(\delta^{i j}+\epsilon^{i l 3} \delta_{l k} \epsilon^{j k 3}\right) I_{i} I_{j}+\delta_{i j} \tilde{I}^{i} \tilde{I}^{j}-2\left(\epsilon^{i p 3}+k \delta^{i p}\right) \delta_{p j} I_{i} \tilde{I}^{j}\right)
$$

with Poisson brackets (see ref. [79] for a derivation)

$$
\begin{aligned}
\left\{I_{i}, I_{j}\right\} & =\epsilon_{i j}{ }^{k} I_{k} \\
\left\{\tilde{I}^{i}, \tilde{I}^{j}\right\} & =f^{i j}{ }_{k} \tilde{I}^{k} \\
\left\{I_{i}, \tilde{I}^{j}\right\} & =\epsilon_{i l}{ }^{j} \tilde{I}^{l}-I_{l} f^{l j}{ }_{i} \quad\left\{\tilde{I}^{i}, I_{j}\right\}=-\epsilon_{j l}{ }^{i} \tilde{I}^{l}+I_{l} f^{l i}{ }_{j}
\end{aligned}
$$

while the Poisson brackets between momenta and configuration space variables $g, \tilde{g}$ are unchanged with respect to $\mathrm{T}^{*} \mathrm{SU}(2), \mathrm{T}^{*} \mathrm{SB}(2, \mathbb{C})$.

In order to derive Hamilton equations, it is sufficient to write in compact form:

$$
\left\{\mathbf{P}_{I}, \mathbf{P}_{J}\right\}=C_{I J}{ }^{K} \mathbf{P}_{K}
$$

with $C_{I J}{ }^{K}$ the $\mathrm{SL}(2, \mathbb{C})$ structure constants as specified above in eqs. (2.52)-(2.54). We have then:

$$
\frac{d}{d t} \mathbf{P}_{I}=\left\{\mathbf{P}_{I}, \widehat{H}\right\}=\left[(\eta+k \mathcal{H})^{-1}\right]^{J K}\left\{\mathbf{P}_{I}, \mathbf{P}_{J}\right\} \mathbf{P}_{K}=\left[(\eta+k \mathcal{H})^{-1}\right]^{J K} C_{I J}{ }^{L} \mathbf{P}_{L} \mathbf{P}_{K}
$$

which is not zero, consistently with (2.48). 
Summarizing,

- we have obtained a dynamical model with doubled coordinates and generalized momenta;

- the Hamiltonian dynamics is dictated by Poisson brackets for the generalized momenta which reproduce the bialgebra structure of $\mathfrak{s l}(2, \mathbb{C})$.

These brackets can be obtained from the following Poisson structure on the double, first introduced in ref. [13]:

$$
\left\{\gamma_{1}, \gamma_{2}\right\}=\frac{\lambda}{2}\left[\gamma_{1}\left(r^{*}-r\right) \gamma_{2}-\gamma_{2}\left(r^{*}-r\right) \gamma_{1}\right]
$$

This is the one that correctly dualizes the bialgebra structure on $\mathfrak{d}$ when evaluated at the identity of the group $D$. To this, let us expand $\gamma \in D$ as $\gamma=\mathbb{1}+i \lambda I_{i} \tilde{e}^{i}+i \lambda \tilde{I}^{i} e_{i}$ and rescale $r, r^{*}$ by the same parameter $\lambda$. It is straightforward to obtain, on the l.h.s. of eq. (2.55), the following expression:

$$
\left\{\gamma_{1}, \gamma_{2}\right\}=-\lambda^{2}\left(\left\{I_{i}, I_{j}\right\} \tilde{e}^{i} \otimes \tilde{e}^{j}+\left\{\tilde{I}^{i}, \tilde{I}^{j}\right\} e_{i} \otimes e_{j}+\left\{I_{i}, \tilde{I}^{j}\right\}\left(\tilde{e}^{i} \otimes e_{j}-e_{j} \otimes \tilde{e}^{i}\right)\right)
$$

while, on the r.h.s. of the same equation, one gets:

$$
-\lambda^{2}\left(I_{s} \epsilon_{i j}^{s} \tilde{e}^{i} \otimes \tilde{e}^{j}+\tilde{I}^{s} f_{s}^{i j} e_{i} \otimes e_{j}+I_{s} f_{i}^{s j}\left(\tilde{e}^{i} \otimes e_{j}-e_{j} \otimes \tilde{e}^{i}\right)+\tilde{I}^{s} \epsilon_{s i}^{j}\left(\tilde{e}^{r} \otimes e_{j}-e_{j} \otimes \tilde{e}^{i}\right)\right) .
$$

By equating the two results, one reproduces the Poisson algebra (2.52)-(2.54), which is the wanted result.

Upon using the compact notation $I=i I_{i} e^{i *}, \tilde{I}=i \tilde{I}^{i} \tilde{e}_{i}^{*}$, with $e^{i^{*}}, \tilde{e}_{i}^{*}$ respectively representing the dual bases of $e_{i}, \tilde{e}^{i}$, one can rewrite the Poisson algebra as follows:

$$
\{I+\tilde{I}, J+\tilde{J}\}=\{I, J\}-\{J, \tilde{I}\}+\{I, \tilde{J}\}+\{\tilde{I}, \tilde{J}\}
$$

which is argued in ref. [79] to represent a Poisson realization of a C-bracket for the generalized bundle $T \oplus T^{*}$ over $\mathrm{SU}(2)$. We refer to ref. [79] for details.

In order to complete the analysis, let us look at the Lie algebra of Hamiltonian vector fields associated with the momenta $I, \tilde{I}$. Hamiltonian vector fields are defined in terms of Poisson brackets in the standard way:

$$
X_{f} \equiv\{\cdot, f\}
$$

so that, by indicating with $X_{i}=\left\{\cdot, I_{i}\right\}, \tilde{X}^{i}=\left\{\cdot, \tilde{I}^{i}\right\}$ the Hamiltonian vector field associated with $I_{i}, \tilde{I}^{i}$ respectively, one has, after using the Jacobi identity, the following Lie algebra:

$$
\begin{aligned}
{\left[X_{i}, X_{j}\right] } & =\left\{\left\{\cdot, I_{j}\right\}, I_{i}\right\}-\left\{\left\{\cdot, I_{i}\right\}, I_{j}\right\}=\left\{\cdot,\left\{I_{i}, I_{j}\right\}\right\}=\epsilon_{i j}{ }^{k}\left\{\cdot, I_{k}\right\}=\epsilon_{i j}{ }^{k} X_{k} \\
{\left[\tilde{X}^{i}, \tilde{X}^{j}\right] } & =\left\{\left\{\cdot, \tilde{I}^{j}\right\}, \tilde{I}^{i}\right\}-\left\{\left\{\cdot, \tilde{I}^{i}\right\}, \tilde{I}^{i}\right\}=\left\{\cdot,\left\{\tilde{I}^{i}, \tilde{I}^{j}\right\}\right\}=f^{i j}{ }_{k}\left\{\cdot, \tilde{I}^{k}\right\}=f^{i j}{ }_{k} \tilde{X}^{k} \\
{\left[X_{i}, \tilde{X}^{j}\right] } & =\left\{\left\{\cdot, \tilde{I}^{j}\right\}, I_{i}\right\}-\left\{\left\{\cdot, I_{i}\right\}, \tilde{I}^{j}\right\}=\left\{\cdot,\left\{I_{i}, \tilde{I}^{j}\right\}\right\}=-f_{i}{ }^{j k}\left\{\cdot, I_{k}\right\}-\left\{\cdot, \tilde{I}^{k}\right\} \epsilon_{k i}{ }^{j} \\
& =-f_{i}{ }^{j k} X_{k}-\tilde{X}^{k} \epsilon_{k i}{ }^{j}
\end{aligned}
$$


namely:

$$
[X+\tilde{X}, Y+\tilde{Y}]=[X, Y]+\mathrm{L}_{X} \tilde{Y}-\mathrm{L}_{Y} \tilde{X}+[\tilde{X}, \tilde{Y}]
$$

which shows that C-brackets can be obtained as derived brackets, in analogy with the ideas of ref.s [93, 94], with the remarkable difference that, in this case, they are derived from the canonical Poisson brackets of the dynamics.

In order to get back one of the two models with half degrees of freedom one has to impose constraints. This has been realized in ref. [79] by gauging the global symmetries of the generalized action, namely the $\mathrm{SU}(2)$ or $\mathrm{SB}(2, \mathbb{C})$ global invariance. The same procedure will be adopted for the chiral model below, therefore we refer again to ref. [79] for details about the gauging of the generalized model described above.

\section{The Principal Chiral Model}

A Principal Chiral Model is a two-dimensional field theory with target configuration space given by a Lie group $G$ and source space given by the two-dimensional spacetime $\mathbb{R}^{1,1}$ endowed with the metric $s_{\alpha \beta}=\operatorname{diag}(1,-1)$.

The SU(2) Principal Chiral Model represents a natural generalization to field theory of the dynamics of the IRR, as described above. Indeed, the action functional is formally the same, while the field variables are defined on two-dimensional spacetime taking values on the group manifold of $\mathrm{SU}(2)$. The possibility of introducing a one-parameter family of Hamiltonian descriptions with modified Poisson brackets, yielding the same equations of motion, was already illustrated in ref.s $[15,16,80,81]$. We are going to follow that approach in order to show that it naturally yields a family of dually related models. The duality transformations which we shall find will be shown to be of Poisson-Lie type.

In the Lagrangian approach the action may be written in terms of fields $\phi:(t, \sigma) \in$ $\mathbb{R}^{1,1} \rightarrow g \in \mathrm{SU}(2)$ and Lie algebra valued left-invariant one-forms whose pull-back to $\mathbb{R}^{1,1}$ may be written as

$$
\phi^{*}\left(g^{-1} \mathrm{~d} g\right)=\left(g^{-1} \partial_{t} g\right) \mathrm{d} t+\left(g^{-1} \partial_{\sigma} g\right) \mathrm{d} \sigma
$$

so to have:

$$
S=\frac{1}{4} \int_{\mathbb{R}^{2}} \operatorname{Tr}\left[\phi^{*}\left(g^{-1} \mathrm{~d} g\right) \wedge \underset{H}{*} \phi^{*}\left(g^{-1} \mathrm{~d} g\right)\right]
$$

where trace is understood as the scalar product in the Lie algebra $\mathfrak{s u}(2)$, and the Hodge star operator acting as $\underset{H}{* \mathrm{~d} t}=\mathrm{d} \sigma, \underset{H}{*} \mathrm{~d} \sigma=\mathrm{d} t,{ }^{5}$ yielding:

$$
S=\frac{1}{4} \int_{\mathbb{R}^{2}} \mathrm{~d} t \mathrm{~d} \sigma \operatorname{Tr}\left[\left\{\left(g^{-1} \partial_{t} g\right)^{2}-\left(g^{-1} \partial_{\sigma} g\right)^{2}\right]\right.
$$

which is to be compared with (2.1) for the IRR dynamics. A remarkable property of the model is that its Euler-Lagrange equations

$$
\partial_{t}\left(g^{-1} \partial_{t} g\right)-\partial_{\sigma}\left(g^{-1} \partial_{\sigma} g\right)=0
$$

\footnotetext{
${ }^{5}$ We adopt the convention $\epsilon_{01}=1$.
} 
may be rewritten in terms of an equivalent system of two first order partial differential equations, introducing the so called currents, as it is customary in the framework of integrable systems:

$$
A^{i}=\operatorname{Tr}\left(g^{-1} \partial_{t} g\right) e_{i}, \quad J^{i}=\operatorname{Tr}\left(g^{-1} \partial_{\sigma} g\right) e_{i},
$$

namely, $g^{-1} \partial_{t} g=2 A^{i} e_{i}, g^{-1} \partial_{\sigma} g=2 J^{i} e_{i}$, with $\operatorname{Tr} e_{i} e_{j}=\frac{1}{2} \delta_{i j}$. The Lagrangian becomes:

$$
L=\frac{1}{2} \int_{\mathbb{R}} \mathrm{d} \sigma\left(A^{i} \delta_{i j} A^{j}-J^{i} \delta_{i j} J^{j}\right)
$$

with

$$
\begin{aligned}
\partial_{t} A & =\partial_{\sigma} J, \\
\partial_{t} J & =\partial_{\sigma} A-[A, J] .
\end{aligned}
$$

The existence of a $g \in \mathrm{SU}(2)$ that admits the expression of the currents in the form (3.5) is guaranteed by eq. (3.8), that can be read as an integrability condition. Moreover, if the usual boundary condition for a physical field is imposed:

$$
\lim _{\sigma \rightarrow \pm \infty} g(\sigma)=1
$$

one has that $g$ is uniquely determined from (3.5). ${ }^{6}$ At fixed $t$, all the elements $g$ satisfying the boundary condition (3.9) form an infinite dimensional Lie group $\mathrm{SU}(2)(\mathbb{R}) \equiv$ $\operatorname{Map}(\mathbb{R}, \mathrm{SU}(2))$, given by smooth maps $g: \sigma \in \mathbb{R} \rightarrow g(\sigma) \in \mathrm{SU}(2)$ which are constant at infinity $[15,16]$. This is a slight generalization of the definition of loop group which is the group of smooth maps from $S^{1}$ to $\mathrm{SU}(2)$.

At fixed time, the currents $J$ and $A$ take values in the Lie algebra $\mathfrak{s u}(2)(\mathbb{R})$, defined as the algebra of functions from $\mathbb{R}$ to $\mathfrak{s u}(2)$ that are sufficiently fast decreasing at infinity to be square-integrable. Again, this definition generalizes the one of loop algebra $\mathfrak{g}\left(S^{1}\right)$, which, for $\mathfrak{g}$ a semi-simple Lie algebra, are known as Kac-Moody algebras.

The analogy with particle dynamics on Lie groups can be pushed further, by regarding the carrier space of the dynamics as the tangent bundle of $\mathrm{SU}(2)(\mathbb{R})$. Therefore the tangent bundle description of the dynamics can be given in terms of $(J, A)$, with $A$ being the left generalized velocities and $J$ playing the role of left configuration space coordinates.

Infinitesimal generators of the Lie algebra $\mathfrak{s u}(2)(\mathbb{R})$ can be obtained by considering the vector fields which generate the finite-dimensional Lie algebra $\mathfrak{s u}(2)$ and replacing ordinary derivatives with functional derivatives, thus yielding

$$
X_{i}(\sigma)=X_{i}^{a}(\sigma) \frac{\delta}{\delta g^{a}(\sigma)}
$$

and their Lie bracket is

$$
\left[X_{i}(\sigma), X_{j}\left(\sigma^{\prime}\right)\right]=c_{i j}^{k} X_{k}(\sigma) \delta\left(\sigma-\sigma^{\prime}\right)
$$

where $\sigma, \sigma^{\prime} \in \mathbb{R}$. This Lie bracket is $C^{\infty}(\mathbb{R})$-linear and $\mathfrak{s u}(2)(\mathbb{R}) \simeq \mathfrak{s u}(2) \otimes C^{\infty}(\mathbb{R})$.

\footnotetext{
${ }^{6}$ Note $[15,16]$ that if we had chosen space to be a circle, (3.8) would not imply (3.5). The solution to these equations will not be periodic in general. If $(A, J)$ is viewed as a connection, (3.8) says that it is flat. But in order for a flat connection to be 'pure gauge' as in (3.5), it is necessary also for the parallel transport operator around a homotopically non-trivial curve (holonomy) to be equal to the identity.
} 
Notice that the real line $\mathbb{R}$ can be replaced by any smooth manifold $M$. The Lie algebras $\mathfrak{g}(M)=\operatorname{Map}(M, \mathfrak{g})$ are the so called current algebras.

\subsection{The Hamiltonian formulation}

Let us briefly review the standard Hamiltonian approach which can be found for example in $[95,96]$. Having recalled in previous section that the target space where the Lagrangian dynamics takes place is the tangent bundle TSU(2), we shall see in present section that in the Hamiltonian framework the target phase space is naturally given by $\mathrm{T}^{*} \mathrm{SU}(2)$. In order to introduce the canonical formalism, the canonical momenta are defined as:

$$
I_{i}=\frac{\delta L}{\delta\left(g^{-1} \partial_{t} g\right)^{i}}=\delta_{i j}\left(g^{-1} \partial_{t} g\right)^{j}=\delta_{i j} A^{j}
$$

Thus, the Hamiltonian can be written as:

$$
H=\frac{1}{2} \int_{\mathbb{R}} \mathrm{d} \sigma\left(I_{i} I_{j} \delta^{i j}+J^{i} J^{j} \delta_{i j}\right)
$$

while the equal-time Poisson brackets $[95,96]$ can be checked to be (see appendix A for a pedagogical derivation)

$$
\begin{aligned}
\left\{I_{i}(\sigma), I_{j}\left(\sigma^{\prime}\right)\right\} & =\epsilon_{i j}{ }^{k} I_{k}(\sigma) \delta\left(\sigma-\sigma^{\prime}\right), \\
\left\{I_{i}(\sigma), J^{j}\left(\sigma^{\prime}\right)\right\} & =\epsilon_{k i}{ }^{j} J^{k}(\sigma) \delta\left(\sigma-\sigma^{\prime}\right)-\delta_{i}^{j} \delta^{\prime}\left(\sigma-\sigma^{\prime}\right), \\
\left\{J^{i}(\sigma), J^{j}\left(\sigma^{\prime}\right)\right\} & =0,
\end{aligned}
$$

yielding the equations of motion for the momenta:

$$
\partial_{t} I_{j}(\sigma)=\left\{H, I_{j}(\sigma)\right\}=\partial_{\sigma} J^{k} \delta_{k j}(\sigma),
$$

where we have used the antisymmetry of the structure constants and the integration by parts. In a similar way, we get the remaining equations:

$$
\partial_{t} J^{j}(\sigma)=\left\{H, J^{j}(\sigma)\right\}=\partial_{\sigma} I_{k} \delta^{k j}(\sigma)-{ }^{j l}{ }_{k} I_{l} J^{k}(\sigma)
$$

The brackets (3.14)-(3.16) show that $I$ and $J$ span the infinite-dimensional current algebra $\mathfrak{c}_{1}$. In particular, the $I$ 's are the generators of the affine Lie algebra $\mathfrak{s u}(2)(\mathbb{R})$, while the $J$ 's span an Abelian algebra $\mathfrak{a}(\mathbb{R})$, so that $\mathfrak{c}_{1}$ is the semi-direct sum $\mathfrak{c}_{1}=\mathfrak{s u}(2)(\mathbb{R}) \ltimes \mathfrak{a}(\mathbb{R})$.

As noticed before, if one extends the analogy with the Lagrangian description of particle dynamics on Lie groups to the Hamiltonian setting, the target phase space of the dynamics can be recognized to be the cotangent bundle of $\mathrm{SU}(2)$, with the currents $\left(J^{i}, I_{i}\right)$ playing the role of conjugate variables and $I$ the left generalized momenta, while $J$ keeping the role of left configuration space coordinates.

A remarkable result due to Rajeev $[15,16]$ consists in the fact that an equivalent description of the dynamics can be given in terms of a new one-parameter family of Poisson 
algebras and modified Hamiltonians. Upon introducing a parameter $\tau$, real or imaginary, the deformed brackets read as:

$$
\begin{aligned}
\left\{I_{i}(\sigma), I_{j}\left(\sigma^{\prime}\right)\right\} & =\left(1-\tau^{2}\right) \epsilon_{i j}{ }^{k} I_{k}(\sigma) \delta\left(\sigma-\sigma^{\prime}\right), \\
\left\{I_{i}(\sigma), J^{j}\left(\sigma^{\prime}\right)\right\} & =\left(1-\tau^{2}\right) J^{k}(\sigma) \epsilon_{k i}{ }^{j} \delta\left(\sigma-\sigma^{\prime}\right)-\left(1-\tau^{2}\right)^{2} \delta_{i}^{j} \delta^{\prime}\left(\sigma-\sigma^{\prime}\right), \\
\left\{J^{i}(\sigma), J^{j}\left(\sigma^{\prime}\right)\right\} & =\left(1-\tau^{2}\right) \tau^{2} \epsilon^{i j}{ }_{k} I_{k}(\sigma) \delta\left(\sigma-\sigma^{\prime}\right) .
\end{aligned}
$$

The modified Hamiltonian reads in turn as:

$$
H_{\tau}=\frac{1}{2\left(1-\tau^{2}\right)^{2}} \int_{\mathbb{R}} \mathrm{d} \sigma\left(I_{i} I_{j} \delta^{i j}+J^{i} J^{j} \delta_{i j}\right)
$$

and, in the limit $\tau \rightarrow 0$, the algebra and the Hamiltonian reduce to the original ones. Notice that the factor $\left(1-\tau^{2}\right)$ is never zero for imaginary $\tau$.

The new brackets correspond to the infinite-dimensional Lie algebra $\mathfrak{c}_{2}$ which, for imaginary $\tau$, our choice from now on, can be easily recognized to be isomorphic to the current algebra modeled on the Lorentz algebra $\mathfrak{s l}(2, \mathbb{C})$, that is $\mathfrak{c}_{2} \simeq \mathfrak{s l}(2, \mathbb{C})(\mathbb{R}) .{ }^{7}$ The Lie algebra $\mathfrak{c}_{1}$ can be recovered in the limit $\tau \rightarrow 0$.

The new equations of motion read then as:

$$
\begin{aligned}
\partial_{t} I_{j}(\sigma) & =\left\{H_{\tau}, I_{j}(\sigma)\right\}=\partial_{\sigma} J^{k} \delta_{k j} \\
\partial_{t} J^{j}(\sigma) & =\left\{H_{\tau}, J^{j}(\sigma)\right\}=\partial_{\sigma} I_{k} \delta^{k j}-\epsilon{ }_{k}{ }_{k} I_{l} J^{k},
\end{aligned}
$$

which coincide with eqs. (3.17), (3.18). Let us notice here that the same deformed algebra, namely the affine Lie algebra of $\mathrm{SL}(2, \mathbb{C})$ or $\mathrm{SO}(4)$, according to $\tau$ being imaginary or real, has been considered in [88] with the main difference that in the latter case the author gets a true deformation of the dynamics, whereas in our case we have an alternative description of one and the same dynamics. As anticipated in the introduction, this should not be surprising, since the cotangent space $\mathrm{T}^{*} \mathrm{SU}(2)$ and the phase space $\mathrm{SL}(2 ; \mathbb{C})$ are symplectomorphic.

Let us rescale the fields according to

$$
\frac{I}{\left(1-\tau^{2}\right)} \rightarrow I \quad \frac{J}{\left(1-\tau^{2}\right)} \rightarrow J
$$

so that the Poisson algebra becomes

$$
\begin{aligned}
\left\{I_{i}(\sigma), I_{j}\left(\sigma^{\prime}\right)\right\} & =\epsilon_{i j}{ }^{k} I_{k}(\sigma) \delta\left(\sigma-\sigma^{\prime}\right), \\
\left\{I_{i}(\sigma), J^{j}\left(\sigma^{\prime}\right)\right\} & =J^{k}(\sigma) \epsilon_{k i}{ }^{j} \delta\left(\sigma-\sigma^{\prime}\right)-\delta_{i}^{j} \delta^{\prime}\left(\sigma-\sigma^{\prime}\right), \\
\left\{J^{i}(\sigma), J^{j}\left(\sigma^{\prime}\right)\right\} & =\tau^{2} \epsilon^{i j}{ }_{k} I_{k}(\sigma) \delta\left(\sigma-\sigma^{\prime}\right)
\end{aligned}
$$

while the rescaled Hamiltonian becomes identical to the undeformed one (3.13). Once identified the Lie algebra here described by the deformed Poisson brackets, one can define

\footnotetext{
${ }^{7}$ For real $\tau$ it is instead isomorphic to the algebra $\mathfrak{s o}(4)(\mathbb{R})$. The latter case is the one analyzed in detail in $[15,16,80,81]$ with respect to quantization and integrability. Here we stick to imaginary $\tau$, this being the choice which unveils the double group structure.
} 
new generators which make it easier to recognize the bi-algebra structure on it. As in the finite dimensional case, we keep the generators of $\mathfrak{s u}(2)(\mathbb{R})$ unmodified and consider the linear combination:

$$
K^{i}(\sigma)=J^{i}(\sigma)-i \tau \epsilon^{l i 3} I_{l}(\sigma) .
$$

From the deformed Poisson brackets (3.26)-(3.28) it is possible to derive the Poisson brackets of the new generators:

$$
\left\{K^{i}(\sigma), K^{j}\left(\sigma^{\prime}\right)\right\}=i \tau \epsilon^{i j l} \epsilon_{l 3 k} K^{k}\left(\sigma^{\prime}\right) \delta\left(\sigma-\sigma^{\prime}\right)
$$

showing that the $K$ 's span the $\mathfrak{s b}(2, \mathbb{C})(\mathbb{R})$ Lie algebra, with structure constants $f^{i j}{ }_{k}=$ $\epsilon^{i j l} \epsilon_{l 3 k}$, while for the mixed Poisson brackets one finds:

$$
\begin{aligned}
& \left\{I_{i}(\sigma), K^{j}\left(\sigma^{\prime}\right)\right\}=\left\{I_{i}(\sigma), J^{j}\left(\sigma^{\prime}\right)-i \tau \epsilon^{j l 3} I_{l}\left(\sigma^{\prime}\right)\right\} \\
& =\left(K^{k}\left(\sigma^{\prime}\right) \epsilon_{k i}{ }^{j}-i \tau I_{k}\left(\sigma^{\prime}\right) \epsilon^{k j s} \epsilon_{s 3 i}\right) \delta\left(\sigma-\sigma^{\prime}\right)-\delta_{i}^{j} \delta^{\prime}\left(\sigma-\sigma^{\prime}\right)
\end{aligned}
$$

where we recognize again the structure constants of the Lie algebra $\mathfrak{s b}(2, \mathbb{C}), \epsilon^{k j s} \epsilon_{s 3 i}=f^{k j}{ }_{i}$. Notice that, in deriving the Poisson algebra above one has to use the Jacobi identity for the structure constants of $\mathrm{SU}(2)$ with one index equal to 3

$$
\epsilon_{q s 3} \epsilon_{s j i}+\epsilon_{i s 3} \epsilon_{q j s}+\epsilon_{j s 3} \epsilon_{i q s}=0
$$

yielding

$$
f^{q i}{ }_{j}=\epsilon^{q i s} \epsilon_{j s 3}=-\epsilon^{q s}{ }_{3} \epsilon_{s j}{ }^{i}-\epsilon^{i s}{ }_{3} \epsilon^{q}{ }_{j s} .
$$

In this way, the Lie algebra $\mathfrak{c}_{2} \equiv \mathfrak{s l}(2, \mathbb{C})(\mathbb{R})$ has been expressed as $\mathfrak{c}_{2}=\mathfrak{s u}(2)(\mathbb{R}) \bowtie$ $\mathfrak{s b}(2, \mathbb{C})(\mathbb{R})$, up to a central extension with central charge equal to -1 , i.e. just like the affine algebra associated with the Drinfel'd double of the Lie algebra $\mathfrak{s u}(2)$ considered at the beginning.

To summarize, upon rewriting the alternative Hamiltonian (3.22) in terms of the new generators, the $\mathrm{SU}(2)$ chiral model is completely described by the one-parameter family of Hamiltonian functions

$$
H_{\tau}=\frac{1}{2} \int_{\mathbb{R}} \mathrm{d} \sigma\left[I_{s} I_{l}\left(\delta_{i}^{s} \delta_{j}^{l}-\tau^{2} \epsilon^{s}{ }_{i 3} \epsilon^{l}{ }_{j 3}\right) \delta^{i j}+K^{i} K^{j} \delta_{i j}+2 i \tau \epsilon^{s l 3} I_{s} K^{q} \delta_{l q}\right]
$$

with Poisson brackets given by:

$$
\begin{aligned}
\left\{I_{i}(\sigma), I_{j}\left(\sigma^{\prime}\right)\right\} & =\epsilon_{i j}{ }^{k} I_{k}(\sigma) \delta\left(\sigma-\sigma^{\prime}\right) \\
\left\{K^{i}(\sigma), K^{j}\left(\sigma^{\prime}\right)\right\} & =i \tau f^{i j}{ }_{k} K^{k}\left(\sigma^{\prime}\right) \delta\left(\sigma-\sigma^{\prime}\right) \\
\left\{I_{i}(\sigma), K^{j}\left(\sigma^{\prime}\right)\right\} & =\left(K^{k}\left(\sigma^{\prime}\right) \epsilon_{k i}{ }^{j}+i \tau f^{j k}{ }_{i} I_{k}\left(\sigma^{\prime}\right)\right) \delta\left(\sigma-\sigma^{\prime}\right)-\delta_{i}^{j} \delta^{\prime}\left(\sigma-\sigma^{\prime}\right)
\end{aligned}
$$

yielding the interesting result that the Principal Chiral Model with compact target space may be described in terms of a non-compact current algebra. This result can be traced back to the fact that the cotangent bundle of the group $\mathrm{SU}(2)$ is symplectomorphic to the group $\mathrm{SL}(2, \mathbb{C})$. We shall see in the next section that this is not the case for the cotangent bundle of the dual group of $\mathrm{SU}(2)$. 
Remarkably, the Hamiltonian (3.33) may be rewritten in terms of a Riemannian metric which we choose to denote as an inverse metric, $\mathcal{H}_{\tau}{ }^{-1}$, for reasons that will be clear in a moment. By introducing:

$$
\mathcal{H}_{\tau}{ }^{-1}=\left(\begin{array}{cc}
h^{i j}(\tau) & i \tau \epsilon^{i p 3} \delta_{p j} \\
i \tau \delta_{i p} \epsilon^{j p 3} & \delta_{i j}
\end{array}\right)
$$

where it has been defined, for future convenience:

$$
h^{i j}(\tau)=\delta^{i j}-\tau^{2} \epsilon^{i a 3} \delta_{a b} \epsilon^{j b 3}
$$

one has indeed:

$$
H_{\tau}=\frac{1}{2} \int_{\mathbb{R}} \mathrm{d} \sigma\left[I_{s} I_{l}\left(\mathcal{H}_{\tau}{ }^{-1}\right)^{s l}+K^{s} K^{l}\left(\mathcal{H}_{\tau}{ }^{-1}\right)_{s l}+K^{s} I_{l}\left(\mathcal{H}_{\tau}{ }^{-1}\right)_{s}{ }^{l}+I_{s} K^{l}\left(\mathcal{H}_{\tau}{ }^{-1}\right)^{s}{ }_{l}\right] .
$$

Let us observe that the metric $\mathcal{H}_{\tau}{ }^{-1}$ coincides with the inverse of $\mathcal{H}$ defined in (2.30) for $\tau=-i$, while $h^{i j}(\tau) \underset{\tau= \pm i}{\rightarrow} h^{i j}$ of eq. (2.33). Moreover one has:

$$
h_{i j}(\tau)=\delta_{i j}+\frac{\tau^{2}}{1-\tau^{2}} \epsilon_{i a 3} \delta^{a b} \epsilon_{j b 3}
$$

with $h_{i j}(\tau)$ the inverse metric of $h^{i j}(\tau)$. In terms of the compact notation $I_{J}=\left(I_{j}, K^{j}\right)$, one can rewrite the Hamiltonian as:

$$
H_{\tau}=\frac{1}{2} \int_{\mathbb{R}} \mathrm{d} \sigma I_{L}\left(\mathcal{H}_{\tau}^{-1}\right)^{L M} I_{M}
$$

Thus, summarizing the results of this section, we have a whole family of models, labelled by the parameter $\tau$, which are related (and indeed equivalent) to the standard $\mathrm{SU}(2)$ chiral model by the linear transformation (3.29), which can be checked to be a $O(3,3)$ transformation. This transformation is a symmetry of the dynamics because it maps solutions into solutions.

\subsubsection{Poisson-Lie structure}

The PCM, in the formulation given by the Hamiltonian in eq. (3.39), together with the Poisson algebra (3.34)-(3.36), is a Poisson-Lie sigma-model according to the following analysis.

Keeping in mind that $K^{i}, I_{i}$ are coordinate functions for the target phase space of the model, $\mathrm{SU}(2) \ltimes \tilde{\mathfrak{g}}$, with $K^{i}$ base coordinates and $I_{i}$ fiber coordinates, we associate to $K^{i}$ the Hamiltonian vector fields (2.57)

$$
X_{K^{i}}:=\left\{\cdot, K^{i}\right\}
$$

spanning the fibers which are isomorphic to the vector space $\mathbb{R}^{3}$. Because of the non-trivial Poisson bracket (3.35), the latter becomes a non-Abelian algebra according to the following (cfr. eqs. (2.58)-(2.60)):

$$
\left[X_{K^{i}}, X_{K^{j}}\right]=X_{\left\{K^{i}, K^{j}\right\}}=i \tau f_{k}^{i j} X_{K^{k}}
$$


Hence, we obtain the dual Lie algebra $\mathfrak{s b}(2, \mathbb{C})$, and in the limit $\tau \rightarrow 0$ we recover the Abelian structure of the starting model over $\mathrm{T}^{*} \mathrm{SU}(2)$. A dual formulation of this property can be given in terms of the Hamiltonian vector fields associated with the currents $I_{i}$, say $X_{i}$. By repeating the analysis above, they can be seen to close the Lie algebra of $\mathfrak{s u}(2)$, hence, they can be regarded as one-forms over the dual Lie algebra, which has become non-Abelian, according to the computation above. We have then:

$$
d X_{i}\left(\tilde{X}^{j}, \tilde{X}^{k}\right)=-X_{i}\left(\left[\tilde{X}^{j}, \tilde{X}^{k}\right]\right)=-f_{i}^{j k}
$$

reproducing, in this way, the commonly used definition of Poisson-Lie structure.

\subsubsection{A family of Born geometries}

We have just seen in section 3.1 how the deformation of the Poisson algebra $\mathfrak{c}_{1}=\mathfrak{s u}(2)(\mathbb{R}) \ltimes \mathfrak{a}$ into $\mathfrak{c}_{2}=\mathfrak{s l}(2, \mathbb{C})$ induces an alternative formulation of the Hamiltonian dynamics of the Principal Chiral Model with target space SU(2). In this formulation we have seen the Riemannian metric $\mathcal{H}_{\tau}^{-1}$ (3.37) emerging in the definition of the alternative Hamiltonian $H_{\tau}(3.39)$.

In order to understand the geometric meaning of such metric, let us take a step back to the original Hamiltonian $H$ (3.13). We can write the undeformed Hamiltonian as

$$
H=\frac{1}{2} \int_{\mathbb{R}} \mathrm{d} \sigma I_{I}\left(\mathcal{H}_{0}^{-1}\right)^{I J} I_{J}
$$

where $I_{I}=\left(I_{i}, J^{i}\right)$ are components of the current 1-form on $\mathrm{T}^{*} \mathrm{SU}(2)$ and

$$
\left(\mathcal{H}_{0}^{-1}\right)^{I J}=\left(\begin{array}{cc}
\delta^{i j} & 0 \\
0 & \delta_{i j}
\end{array}\right)
$$

is a Riemannian metric on $\mathrm{T}^{*} \mathrm{SU}(2)$. In other words, the Hamiltonian description of the Principal Chiral Model on SU(2) naturally involves the Riemannian metric $\mathcal{H}_{0}^{-1}$ on the cotangent bundle.

Interestingly, the metric (3.46) can be interpreted as one of the structures defining a left-invariant Born geometry on $\mathrm{T}^{*} \mathrm{SU}(2)$. In the present case the transformation defining a Born geometry, as detailed below, acts as an $O(3,3)$ transformation of the target phase $\mathrm{T}^{*} \mathrm{SU}(2)$.

The concept of Born reciprocity giving rise to Born geometries has been first introduced, up to our knowledge, by Freidel and collaborators in [83], in order to provide a new point of view on string theory in which spacetime is a derived dynamical concept. We shall see that the family of models which we have described in the previous section can be related with such interpretation, with the phase space of the chiral model regarded as dynamical. Born reciprocity is thus implemented as a choice of a Lagrangian submanifold of the phase space, in our case governed by the parameter $\tau$, and amounts to a generalization of T-duality. In this approach the phase space of the model can be understood in terms of dynamical bi-Lagrangian manifolds whose geometric structure is an example of a Born 
geometry. Let us notice that, in our case, such a bi-Lagrangian manifold happens to be a Drinfel'd double as well, with an interesting overlap between the two structures.

To this, let us start by recalling that $\mathrm{T}^{*} \mathrm{SU}(2)$ is a Drinfel'd double with Lie algebra $\mathfrak{s u}(2) \ltimes \mathbb{R}^{3}$. Such Lie algebra has a natural (symmetric, non-degenerate) pairing $\langle\cdot, \cdot\rangle$ such that $\mathfrak{s u}(2)$ and $\mathbb{R}^{3}$ are maximally isotropic subspaces with respect to it. Moreover, $\mathfrak{s u}(2) \ltimes \mathbb{R}^{3}$ can be seen as a split vector space $\mathfrak{s u}(2) \oplus \mathbb{R}^{3}$, thus it can be naturally endowed with a para-complex structure $\kappa$, i.e. $\kappa \in \operatorname{End}\left(\mathfrak{s u}(2) \ltimes \mathbb{R}^{3}\right)$ such that $\kappa^{2}=\mathbb{1}$ with $\mathfrak{s u}(2)$ eigenspace of $\kappa$ associated with the eigenvalue +1 and $\mathbb{R}^{3}$ eigenspace associated with the eigenvalue -1 . The structures $\langle\cdot, \cdot\rangle$ and $\kappa$ satisfy a compatibility condition

$$
\langle\kappa(\xi), \psi\rangle=-\langle\kappa(\psi), \xi\rangle, \quad \forall \xi, \psi \in \mathfrak{s u}(2) \ltimes \mathbb{R}^{3},
$$

which defines a two-form $\omega$ on $\mathfrak{s u}(2) \ltimes \mathbb{R}^{3}$. Summarizing, $(\langle\cdot, \cdot\rangle, \kappa)$ related by the above compatibility condition define a para-Hermitian structure on $\mathfrak{s u}(2) \ltimes \mathbb{R}^{3}$.

Since $T\left(\mathrm{~T}^{*} \mathrm{SU}(2)\right) \cong \mathrm{T}^{*} \mathrm{SU}(2) \times\left(\mathfrak{s u}(2) \ltimes \mathbb{R}^{3}\right)$, we may read the structures $(\langle\cdot, \cdot\rangle, \kappa)$ as defined pointwise on $\mathrm{T}^{*} \mathrm{SU}(2)$, giving, respectively, a left-invariant $O(3,3)$ metric $\eta$ and an endomorphism $\kappa$ of $T\left(\mathrm{~T}^{*} \mathrm{SU}(2)\right)$ such that $\kappa^{2}=\mathbb{1}$ which has TSU(2) (eigenvalue +1$)$ and $T \mathbb{R}^{3}$ (eigenvalue -1 ) as eigenbundles ${ }^{8}$ (with a slight abuse of notation for $\kappa$ ). Again, one has a so-called fundamental two-form $\omega=\eta \kappa$ on $\mathrm{T}^{*} \mathrm{SU}(2)$ coming from the compatibility of $\eta$ and $\kappa$.

In order to understand the relation between the left-invariant para-Hermitian structure $(\eta, \kappa)$ and the Riemannian metric (3.46), let us consider the (global) basis $\left\{\alpha^{i}, \varphi_{i}\right\}$ of leftinvariant 1-forms on $\mathrm{T}^{*} \mathrm{SU}(2)$ with dual left-invariant vector fields $\left\{X_{i}, Y^{i}\right\}$. Then, the para-Hermitian structure $(\eta, \kappa)$ on $\mathrm{T}^{*} \mathrm{SU}(2)$ can be written as

$$
\begin{aligned}
& \eta=\alpha^{i} \otimes \varphi_{i}+\varphi_{i} \otimes \theta^{i}, \\
& \kappa=X_{i} \otimes \alpha^{i}-Y^{i} \otimes \varphi_{i} .
\end{aligned}
$$

The fundamental two-form then reads as:

$$
\omega=\eta \kappa=\varphi_{i} \wedge \alpha^{i}
$$

and the Riemannian metric (3.46) is written as

$$
\mathcal{H}_{0}=\delta^{i j} \varphi_{i} \otimes \varphi_{j}+\delta_{i j} \alpha^{i} \otimes \alpha^{j} .
$$

Note that the left-invariant Riemannian metric $\mathcal{H}_{0}$ is the unique left-invariant metric such that left-invariant vector fields are orthonormal. So its appearance in the Hamiltonian is

\footnotetext{
${ }^{8}$ One may also say that $\pi: \mathrm{T}^{*} \mathrm{SU}(2) \rightarrow \mathrm{SU}(2)$ is foliated by $\mathrm{SU}(2)$ and $\mathbb{R}^{3}$. Note that, in this case, the foliation $\mathbb{R}^{3}$ can be also seen as given by the canonical vertical subbundle $V=k e r(d \pi)$ of $T\left(\mathrm{~T}^{*} \mathrm{SU}(2)\right)$ defined as the kernel of $\mathrm{d} \pi: T\left(\mathrm{~T}^{*} \mathrm{SU}(2)\right) \rightarrow \mathrm{TSU}(2)$. The other foliation may be obtained by a choice of the horizontal distribution such that horizontal vectors are left-invariant with respect to $\mathrm{SU}(2)$, i.e. splitting the canonical short exact sequence

$$
0 \rightarrow V \rightarrow T\left(\mathrm{~T}^{*} \mathrm{SU}(2)\right) \rightarrow \pi^{\star}(\mathrm{TSU}(2)) \rightarrow 0
$$

with the proper horizontal lift of left-invariant vector fields. A para-complex structure is naturally associated with such splitting.
} 
completely natural in the context of Lie groups. Therefore, from the above expressions it is easy to verify that:

$$
\eta^{-1} \mathcal{H}_{0}=\mathcal{H}_{0}^{-1} \eta \quad \omega^{-1} \mathcal{H}_{0}=-\mathcal{H}_{0}^{-1} \omega
$$

These are the defining relations for the Born structure $\left(\eta, \kappa, \mathcal{H}_{0}\right)$ on $\mathrm{T}^{*} \mathrm{SU}(2)$. This is the canonical Born geometry induced by the Drinfel'd double structure, see [83, 87] for details.

The deformed Hamiltonian $H_{\tau}$ also gives a Riemannian metric on $\mathrm{T}^{*} \mathrm{SU}(2)$ and we shall see that such metric $\mathcal{H}_{\tau}$ is a $B$-transformation of the metric $\mathcal{H}_{0}$.

Let us consider the $\tau$-dependent $B$-transformation

$$
e^{B(\tau)}=\left(\begin{array}{cc}
\mathbb{1} & i \tau B \\
0 & \mathbb{1}
\end{array}\right) \in O(3,3)
$$

such that the components of the tensor $B$ are given by $B^{i j}=\epsilon^{i j 3}$

The Riemannian metric $\mathcal{H}_{\tau}$, inverse of (3.37), is obtained by the $B$-transformation acting on $\mathcal{H}_{0}$ :

$$
\mathcal{H}_{\tau}=\left(e^{-B(\tau)}\right)^{t} \mathcal{H}_{0} e^{B(\tau)},
$$

i.e. it has components:

$$
\left(\mathcal{H}_{\tau}\right)_{I J}=\left(\begin{array}{cc}
\delta_{i j} & i \tau \delta_{i p} \epsilon^{j p 3} \\
i \tau \epsilon^{i p 3} \delta_{p j} & \delta^{i j}-\tau^{2} \epsilon^{i s 3} \delta_{s l} \epsilon^{j l 3}
\end{array}\right) .
$$

Furthermore, the left-invariant para-Hermitian structure $(\eta, \kappa)$ is transformed under $e^{B(\tau)}$. In particular, the only structure which changes under such transformation is the para-complex structure $\kappa$, i.e.

$$
\kappa_{\tau}=e^{B(\tau)} \kappa e^{-B(\tau)}
$$

with $\kappa_{\tau}$ still compatible with $\eta$, so that the fundamental two-form becomes $\omega_{\tau}=\eta \kappa_{\tau}$. In matrix form, the new almost para-Hermitian structure reads as:

$$
\kappa_{\tau}=\left(\begin{array}{cc}
\mathbb{1} & 2 i \tau B \\
0 & \mathbb{1}
\end{array}\right) \quad \eta=\left(\begin{array}{ll}
0 & \mathbb{1} \\
\mathbb{1} & 0
\end{array}\right) \quad \omega_{\tau}=\left(\begin{array}{lc}
0 & \mathbb{1} \\
\mathbb{1} & 2 i \tau \eta B
\end{array}\right)
$$

where $\eta B \in \Gamma\left(\wedge^{2} T^{*} \mathbb{R}^{3}\right)$. Note that the new almost para-Hermitian structure still has TSU(2) as eigenbundle while $T \mathbb{R}^{3}$ is transformed in a non-involutive distribution $V_{\tau}$ whose sections are generated by vector fields in the form $\bar{Y}^{i}=Y^{i}+i \tau \epsilon^{i j 3} X_{j}$. We can easily check that the metric $\mathcal{H}_{\tau}$ gives a Born structure on $\mathrm{T}^{*} \mathrm{SU}(2)$ together with $\left(\eta, \kappa_{\tau}\right)$, for each value of the parameter $\tau$.

We finally show that the deformed current algebra defined in eqs. (3.34)-(3.36) is obtained via the same $B$-transformation of the Poisson current algebra of the fields $J^{i}, I_{i}$. The latter can be stated in terms of the Poisson bivector field:

$$
\Lambda=\int \mathrm{d} \sigma \mathrm{d} \sigma^{\prime} \Lambda^{I J}\left(\sigma, \sigma^{\prime}\right) X_{I}(\sigma) \wedge X_{J}\left(\sigma^{\prime}\right)
$$


with

$$
\begin{aligned}
& \Lambda_{i j}\left(\sigma, \sigma^{\prime}\right)=\epsilon_{i j}{ }^{k} I_{k} \delta\left(\sigma-\sigma^{\prime}\right) \\
& \Lambda^{i j}\left(\sigma, \sigma^{\prime}\right)=0 \\
& \Lambda_{i}{ }^{j}\left(\sigma, \sigma^{\prime}\right)=J^{k}(\sigma) \epsilon_{k i}{ }^{j} \delta\left(\sigma-\sigma^{\prime}\right)-\delta_{i}{ }^{j} \partial_{\sigma} \delta\left(\sigma-\sigma^{\prime}\right) \\
& \Lambda^{i}{ }_{j}\left(\sigma, \sigma^{\prime}\right)=-J^{k}(\sigma) \epsilon_{k j}{ }^{i} \delta\left(\sigma-\sigma^{\prime}\right)+\delta^{i}{ }_{j} \partial_{\sigma} \delta\left(\sigma-\sigma^{\prime}\right) .
\end{aligned}
$$

Thus, the $B$-transformed Poisson structure reads as:

$$
\Lambda_{\tau}^{\prime}=e^{B(\tau)} \Lambda\left(e^{-B(\tau)}\right)^{t}
$$

namely

$$
\Lambda_{\tau}^{\prime I J}=\left(\begin{array}{cc}
\delta^{i}{ }_{j} i \tau B^{i j} \\
0 & \delta_{i}{ }^{j}
\end{array}\right)\left(\begin{array}{cc}
0 & \Lambda^{j}{ }_{k} \\
\Lambda_{j}{ }^{k} & \Lambda_{j k}
\end{array}\right)\left(\begin{array}{cc}
\delta_{k}{ }^{l} & 0 \\
-i \tau B^{k l} & \delta^{k}{ }_{l}
\end{array}\right)
$$

so that we may read the $B$-transformation $e^{B(\tau)}$ as a Poisson map between $\Lambda$ and $\Lambda_{\tau}^{\prime}$, both bivector fields on $\mathrm{T}^{*} \mathrm{SU}(2)$. Note that $\Lambda$ is the Poisson structure on $\mathrm{T}^{*} \mathrm{SU}(2)$ obtained from the canonical (left-invariant) symplectic structure, as shown in appendix A. If we simultaneously rotate the fields according to

$$
\left(\begin{array}{c}
J^{\prime i} \\
I_{i}^{\prime}
\end{array}\right)=\left(\begin{array}{cc}
\delta^{i}{ }_{j} & i \tau B^{i j} \\
0 & \delta_{i}{ }^{j}
\end{array}\right)\left(\begin{array}{l}
J^{j} \\
I_{j}
\end{array}\right)
$$

which is nothing but the $O(3,3)$ transformation (3.29), we reproduce the current algebra of the fields $I, K$, i.e. (3.34), (3.35) and (3.36), upon identifying $J^{\prime}$ with $K$.

Finally, performing the transformations (3.51), (3.62) on the Hamiltonian (3.45) we recover the expression (3.41).

Therefore we can conclude by saying that the family of equivalent Hamiltonian descriptions of the SU(2) PCM, first found in [15, 16], can be understood in terms of a one-parameter family of Born geometries for the target phase space $T^{*} S^{3}$, corresponding, for each choice of the parameter $\tau$, to a specific splitting of phase space, with the value $\tau=0$ the canonical splitting.

\section{Poisson-Lie dual models}

From the Hamiltonian formulation of the $\mathrm{SU}(2)$ chiral model we have seen that it is possible to describe the dynamics in terms of the centrally extended current algebra $\mathfrak{c}_{2}=\mathfrak{s l}(2, \mathbb{C})(\mathbb{R})$. Therefore, as we have done for the rigid rotor, we shall look for a model whose target space is the dual group of $\mathrm{SU}(2)$. As previously anticipated, we shall see that the duality relation between the two models defined on the manifold of Poisson-Lie dual groups, is much more natural in the context of field theory. To this, it is worth recalling that the model described above is a Poisson-Lie sigma model, as we have shown in section 3.1.1.

Let us consider the Poisson algebra $\mathfrak{c}_{2}$ (with central extension), represented by eqs. (3.34)-(3.36) and let us introduce another imaginary parameter $\alpha$ in such a way to 
make the role of the subalgebras $\mathfrak{s u}(2)(\mathbb{R})$ and $\mathfrak{s b}(2, \mathbb{C})(\mathbb{R})$ symmetric. We consider namely the two-parameters generalization of the algebra (3.34)-(3.36)

$$
\begin{aligned}
\left\{I_{i}(\sigma), I_{j}\left(\sigma^{\prime}\right)\right\} & =i \alpha \epsilon_{i j}{ }^{k} I_{k}(\sigma) \delta\left(\sigma-\sigma^{\prime}\right) \\
\left\{K^{i}(\sigma), K^{j}\left(\sigma^{\prime}\right)\right\} & =i \tau f^{i j}{ }_{k} K^{k}\left(\sigma^{\prime}\right) \delta\left(\sigma-\sigma^{\prime}\right) \\
\left\{I_{i}(\sigma), K^{j}\left(\sigma^{\prime}\right)\right\} & =\left(i \alpha K^{k}\left(\sigma^{\prime}\right) \epsilon_{k i}{ }^{j}+i \tau f^{j k}{ }_{i} I_{k}\left(\sigma^{\prime}\right)\right) \delta\left(\sigma-\sigma^{\prime}\right)-\delta_{i}^{j} \delta^{\prime}\left(\sigma-\sigma^{\prime}\right)
\end{aligned}
$$

which, in the limit $i \tau \rightarrow 0$, reproduces the semi-direct sum $\mathfrak{s u}(2)(\mathbb{R}) \ltimes \mathfrak{a}$, while the limit $i \alpha \rightarrow 0$ yields $\mathfrak{s} \mathfrak{b}(2, \mathbb{C})(\mathbb{R}) \ltimes \mathfrak{a}$. For all non zero values of the two parameters, the algebra is isomorphic to $\mathfrak{c}_{2}$, and, upon suitably rescaling the fields, one gets a two-parameter family of models, all equivalent to the Principal Chiral Model.

Since the result might appear surprising at a first sight, let us show in detail how it works, by slightly generalizing the procedure of subsection 3.1. The goal is to show that the dynamics that is derived from the algebra (4.1)-(4.3), together with a suitable Hamiltonian, is equivalent to the dynamics that follows from eqs. (3.14)-(3.16) with the Hamiltonian (3.13). As an intermediate step, one has to rescale the fields $I$ and $K$ as follows:

$$
\bar{I}_{j}=\frac{I_{j}}{i \alpha} \quad \bar{K}^{j}=i \alpha K^{j}
$$

which yield

$$
\begin{aligned}
\left\{\bar{I}_{i}(\sigma), \bar{I}_{j}\left(\sigma^{\prime}\right)\right\} & =\epsilon_{i j}{ }^{k} \bar{I}_{k}(\sigma) \delta\left(\sigma-\sigma^{\prime}\right) \\
\left\{\bar{K}^{i}(\sigma), \bar{K}^{j}\left(\sigma^{\prime}\right)\right\} & =(i \tau i \alpha) f^{i j} \bar{K}^{k}\left(\sigma^{\prime}\right) \delta\left(\sigma-\sigma^{\prime}\right) \\
\left\{\bar{I}_{i}(\sigma), \bar{K}^{j}\left(\sigma^{\prime}\right)\right\} & =\left(\bar{K}^{k}\left(\sigma^{\prime}\right) \epsilon_{k i}{ }^{j}+(i \tau i \alpha) f_{i}^{j k}{ }_{i} \bar{I}_{k}\left(\sigma^{\prime}\right)\right) \delta\left(\sigma-\sigma^{\prime}\right)-\delta_{i}^{j} \delta^{\prime}\left(\sigma-\sigma^{\prime}\right) .
\end{aligned}
$$

The latter is identical to the algebra (3.34)-(3.36), upon introducing $\bar{\tau}$, s.t. $i \bar{\tau}=i \tau i \alpha$. Then we rescale and rotate the fields, analogously to what has been previously done, according to:

$$
\hat{I}_{i}=\left(1-\alpha^{2} \tau^{2}\right) \frac{I_{i}}{i \alpha} \quad \hat{J}^{i}=\left(1-\alpha^{2} \tau^{2}\right)\left(i \alpha K^{i}+i \tau \epsilon^{l i 3} I_{l}\right)
$$

so that the latter obey the Poisson algebra

$$
\begin{aligned}
\left\{\hat{I}_{i}(\sigma), \hat{I}_{j}\left(\sigma^{\prime}\right)\right\} & =\left(1-\alpha^{2} \tau^{2}\right) \epsilon_{i j}{ }^{k} \bar{I}_{k}(\sigma) \delta\left(\sigma-\sigma^{\prime}\right), \\
\left\{\hat{I}_{i}(\sigma), \hat{J}^{j}\left(\sigma^{\prime}\right)\right\} & =\left(1-\alpha^{2} \tau^{2}\right) J^{k}(\sigma) \epsilon_{k i}{ }^{j} \delta\left(\sigma-\sigma^{\prime}\right)-\left(1-\alpha^{2} \tau^{2}\right)^{2} \delta_{i}^{j} \delta^{\prime}\left(\sigma-\sigma^{\prime}\right), \\
\left\{\hat{J}^{i}(\sigma), \hat{J}^{j}\left(\sigma^{\prime}\right)\right\} & =\left(1-\alpha^{2} \tau^{2}\right) \tau^{2} \epsilon^{i j}{ }_{k} \hat{I}_{k}(\sigma) \delta\left(\sigma-\sigma^{\prime}\right)
\end{aligned}
$$

that, together with the modified Hamiltonian

$$
H_{\tau, \alpha}=\frac{1}{2\left(1-\alpha^{2} \tau^{2}\right)^{2}} \int_{\mathbb{R}} \mathrm{d} \sigma\left(\hat{I}_{i} \hat{I}_{j} \delta^{i j}+\hat{J}^{i} \hat{J}^{j} \delta_{i j}\right)
$$

can be checked to yield the equations of motion

$$
\begin{aligned}
\partial_{t} \hat{I}_{j}(\sigma) & =\left\{H_{\tau, \alpha}, \hat{I}_{j}(\sigma)\right\}=\partial_{\sigma} \hat{J}^{k} \delta_{k j} \\
\partial_{t} \hat{J}^{j}(\sigma) & =\left\{H_{\tau, \alpha} J^{j}(\sigma)\right\}=\partial_{\sigma} I_{k} \delta^{k j}-\epsilon{ }_{k}{ }_{k} I_{l} J^{k},
\end{aligned}
$$


namely, the undeformed dynamics of the PCM as in eqs. (3.17), (3.18). In the limit $i \bar{\tau} \rightarrow 0$, the algebra and the Hamiltonian reduce to the original ones. Notice that the factor $1-\alpha^{2} \tau^{2}=1-\bar{\tau}^{2}$ is never zero for imaginary $\bar{\tau}$.

Once we have shown how to recast the two-parameter algebra (4.1)-(4.3) in the form (4.9)-(4.11), it is useful to express the Hamiltonian (4.12) in terms of the fields $I, K$. We get:

$$
H_{\tau, \alpha}=\frac{1}{2} \int_{\mathbb{R}} \mathrm{d} \sigma\left[I_{s} I_{l}\left(\mathcal{H}_{\tau, \alpha}{ }^{-1}\right)^{s l}+K^{s} K^{l}\left(\mathcal{H}_{\tau, \alpha}{ }^{-1}\right)_{s l}+K^{s} I_{l}\left(\mathcal{H}_{\tau, \alpha}{ }^{-1}\right)_{s}{ }^{l}+I_{s} K^{l}\left(\mathcal{H}_{\tau, \alpha}{ }^{-1}\right)^{s}{ }_{l}\right],
$$

with

$$
\mathcal{H}_{\tau, \alpha}{ }^{-1}=\left(\begin{array}{cc}
\frac{h^{i j}(\bar{\tau})}{(i \alpha)^{2}} & i \bar{\tau} \epsilon^{i p 3} \delta_{p j} \\
i \bar{\tau} \delta_{i p} \epsilon^{j p 3} & (i \alpha)^{2} \delta_{i j}
\end{array}\right)
$$

and $i \bar{\tau}$ previously defined. In terms of the compact notation $I_{J}=\left(I_{j}, K^{j}\right)$, one can rewrite the Hamiltonian as:

$$
H_{\tau \alpha}=\frac{1}{2} \int_{\mathbb{R}} \mathrm{d} \sigma I_{L}\left(\mathcal{H}_{\tau, \alpha}^{-1}\right)^{L M} I_{M} .
$$

Since the role of $I$ and $K$ is now symmetric, we can perform an $O(3,3)$ transformation which exchanges the momenta $I_{i}$ with the fields $K^{i}$, thus obtaining a new two-parameter family of models, which legitimately deserve to be called duals to the PCM.

The $O(3,3)$ transformation

$$
\tilde{K}(\sigma)=I(\sigma), \quad \tilde{I}(\sigma)=K(\sigma)
$$

yields, when applied to the Hamiltonian (3.39), the new Hamiltonian

$$
\tilde{H}_{\tau, \alpha}=\frac{1}{2} \int_{\mathbb{R}} \mathrm{d} \sigma\left[\tilde{K}_{s}\left(\mathcal{H}_{\tau, \alpha}{ }^{-1}\right)^{s l} \tilde{K}_{l}+\tilde{I}^{s}\left(\mathcal{H}_{\tau, \alpha}{ }^{-1}\right)_{s l} \tilde{I}^{l}+2 i\left(\mathcal{H}_{\tau, \alpha}{ }^{-1}\right)^{s}{ }_{l} \tilde{K}_{s} \tilde{I}^{l}\right]
$$

with Poisson algebra:

$$
\begin{aligned}
\left\{\tilde{K}_{i}(\sigma), \tilde{K}_{j}\left(\sigma^{\prime}\right)\right\} & =i \alpha \epsilon_{i j}{ }^{k} \tilde{K}_{k}(\sigma) \delta\left(\sigma-\sigma^{\prime}\right) \\
\left\{\tilde{I}^{i}(\sigma), \tilde{I}^{j}\left(\sigma^{\prime}\right)\right\} & =i \tau f^{i j}{ }_{k} \tilde{I}^{k}\left(\sigma^{\prime}\right) \delta\left(\sigma-\sigma^{\prime}\right) \\
\left\{\tilde{K}_{i}(\sigma), \tilde{I}^{j}\left(\sigma^{\prime}\right)\right\} & =\left(i \alpha \tilde{I}^{k}\left(\sigma^{\prime}\right) \epsilon_{k i}{ }^{j}+i \tau f^{j k}{ }_{i} \tilde{K}_{k}\left(\sigma^{\prime}\right)\right) \delta\left(\sigma-\sigma^{\prime}\right)-\delta_{i}^{j} \delta^{\prime}\left(\sigma-\sigma^{\prime}\right) .
\end{aligned}
$$

The Hamiltonian can be recast into the form

$$
\tilde{H}_{\tau}=\frac{1}{2} \int_{\mathbb{R}} \mathrm{d} \sigma \tilde{I}_{I}\left(\mathcal{H}_{\tau, \alpha}\right)^{I J} \tilde{I}_{J}
$$

with $\tilde{I}_{J}=\left(\tilde{I}^{j}, \tilde{K}_{j}\right)$.

From the Poisson algebra (4.20)-(4.22) we observe that the new family of models, which we call DPCM (Dual Principal Chiral Models), has target configuration space the group manifold of $\operatorname{SB}(2, \mathbb{C})$, spanned by the fields $\tilde{K}_{i}$, and momenta $\tilde{I}^{i}$ which span the fibers of the target phase space.

In strict analogy with what we have found previously, we could repeat step by step the analysis performed in section 3.1.1 and conclude that the DPCM are Poisson-Lie sigma 
models according to the definition we have given. Moreover, the two families are dual to each other by construction.

To conclude this section, let us observe that, in the limit $\alpha \rightarrow 0$ the dual current algebra collapses to the semidirect $\operatorname{sum} \mathfrak{s} \mathfrak{b}(2, \mathbb{C})(\mathbb{R}) \ltimes \mathfrak{a}$, but the Hamiltonian (4.23) becomes singular.

In the next section we will approach the problem from a Lagrangian perspective, starting directly with a natural action defined on the Poisson-Lie dual of SU(2).

\subsection{The Lagrangian approach}

Following the approach that we have already used for the rigid rotor, it is natural, within the Lagrangian approach, to introduce fields $\tilde{g}:(t, \sigma) \rightarrow \mathrm{SB}(2, \mathbb{C})$ and one-forms valued in the Lie algebra $\mathfrak{s} \mathfrak{b}(2, \mathbb{C})$, in terms of which a natural Lagrangian can be defined on the Lie-Poisson dual to $\mathrm{SU}(2)$. The Hamiltonian will then be obtained by Legendre transform, together with a Poisson algebra which, not surprisingly, will result to be isomorphic to $\mathfrak{c}_{3}=\mathfrak{s} \mathfrak{b}(2, \mathbb{C})(\mathbb{R}) \ltimes \mathfrak{a}$. This new Hamiltonian will be related to the two-parameter family of dual models introduced above, through a $B$-transformation.

Let us look at the Lagrangian approach in some detail.

The action of the proposed model is a straightforward extension of the one in eq. (2.32) to fields $\tilde{\phi}:(t, \sigma) \in \mathbb{R}^{1,1} \rightarrow \tilde{g} \in \mathrm{SB}(2, \mathbb{C})$, with Lie algebra valued left-invariant one-forms $\tilde{g}^{-1} \mathrm{~d} \tilde{g}$ whose pull-back to $\mathbb{R}^{1,1}$ is given by:

$$
\tilde{\phi}^{*}\left(\tilde{g}^{-1} \mathrm{~d} \tilde{g}\right)=\left(\tilde{g}^{-1} \partial_{t} \tilde{g}\right)_{i} \tilde{e}^{i} \mathrm{~d} t+\left(\tilde{g}^{-1} \partial_{\sigma} \tilde{g}\right)_{i} \tilde{e}^{i} \mathrm{~d} \sigma .
$$

We have then:

$$
\tilde{S}=\frac{1}{2} \int_{\mathbb{R}^{1,1}} \mathcal{T r}\left[\phi^{*}\left(\tilde{g}^{-1} \mathrm{~d} \tilde{g}\right) \wedge \underset{H}{*} \phi^{*}\left(\tilde{g}^{-1} \mathrm{~d} \tilde{g}\right)\right],
$$

where, as in the finite-dimensional case, $\mathcal{T} r$ stands for the non-degenerate product in the Lie algebra $\mathfrak{s} \mathfrak{b}(2, \mathbb{C})$, given by $(2.30)$, and the Hodge star operator acts as $\underset{H}{* \mathrm{~d} t}=\mathrm{d} \sigma, \underset{H}{* \mathrm{~d}} \sigma=\mathrm{d} t$, yielding

$$
\tilde{S}=\frac{1}{2} \int_{\mathbb{R}^{2}} \mathrm{~d} t \mathrm{~d} \sigma\left[\left(\tilde{g}^{-1} \partial_{t} \tilde{g}\right)_{i}\left(\tilde{g}^{-1} \partial_{t} \tilde{g}\right)_{j}-\left(\tilde{g}^{-1} \partial_{\sigma} g\right)_{i}\left(\tilde{g}^{-1} \partial_{\sigma} g\right)_{j}\right] h^{i j}
$$

As for the finite-dimensional case, the action functional is invariant under left $\operatorname{SB}(2, \mathbb{C})$ action. The Euler-Lagrange equations

$$
h^{i j}\left(\partial_{t}\left(\tilde{g}^{-1} \partial_{t} \tilde{g}\right)_{j}-\partial_{\sigma}\left(\tilde{g}^{-1} \partial_{\sigma} \tilde{g}\right)_{j}\right)=\mathrm{L}_{\tilde{X}^{i}} \tilde{L}
$$

with $\tilde{X}^{i}(\sigma)$ the left-invariant vector fields over the group manifold and $\tilde{L}$ the Lagrangian, may be rewritten in terms of an equivalent system of two first order partial differential equations, introducing, as for the $\mathrm{SU}(2)$ principal model, the currents: ${ }^{9}$

$$
\tilde{A}_{i}=\left(\tilde{g}^{-1} \partial_{t} \tilde{g}\right)_{i}, \quad \tilde{J}_{i}=\left(\tilde{g}^{-1} \partial_{\sigma} \tilde{g}\right)_{i} .
$$

\footnotetext{
${ }^{9}$ No factor two is needed here because $\mathcal{T} r\left(\tilde{e}^{i} \tilde{e}^{j}\right)=\delta^{i j}$.
} 
The Lagrangian becomes then:

$$
\tilde{L}=\frac{1}{2} \int_{\mathbb{R}} \mathrm{d} \sigma\left(\tilde{A}_{i} h^{i j} \tilde{A}_{j}-\tilde{J}_{i} h^{i j} \tilde{J}_{k}\right)
$$

and the equations of motion read:

$$
\begin{aligned}
h^{i j}\left(\partial_{t} \tilde{A}_{j}-\partial_{\sigma} \tilde{J}_{j}\right) & =f_{l}^{s i} h^{l j}\left(\tilde{A}_{s} \tilde{A}_{j}-\tilde{J}_{s} \tilde{J}_{j}\right), \\
\partial_{t} \tilde{J} & =\partial_{\sigma} \tilde{A}-[\tilde{A}, \tilde{J}],
\end{aligned}
$$

being the latter a condition for the existence of $\tilde{g} \in \mathrm{SB}(2, \mathbb{C})$ that admits the expression of the currents in the form (4.28). At fixed $t$, all elements $\tilde{g}$ satisfying the boundary condition $\lim _{\sigma \rightarrow \pm \infty} g(\sigma)=1$ form the infinite-dimensional Lie group $\operatorname{SB}(2, \mathbb{C})(\mathbb{R}) \equiv \operatorname{Map}(\mathbb{R}, \operatorname{SB}(2, \mathbb{C}))$, given by smooth maps $\tilde{g}: \sigma \in \mathbb{R} \rightarrow \tilde{g}(\sigma) \in \mathrm{SB}(2, \mathbb{C})$ which are constant at infinity.

At fixed time, the currents $\tilde{J}$ and $\tilde{A}$ take values in the Lie algebra $\mathfrak{s} \mathfrak{b}(2, \mathbb{C})(\mathbb{R})$ of functions from $\mathbb{R}$ to $\mathfrak{s b}(2, \mathbb{C})$ that are sufficiently fast decreasing at infinity to be squareintegrable. Therefore the tangent bundle description of the dual dynamics can be given in terms of $(\tilde{J}, \tilde{A})$, with $\tilde{A}$ the left generalized velocities, while $\tilde{J}$ playing the role of left configuration space coordinates.

\subsubsection{The Hamiltonian description}

Upon introducing left momenta

$$
\tilde{I}^{i}=\frac{\delta \tilde{L}}{\delta\left(\tilde{g}^{-1} \partial_{t} \tilde{g}\right)_{i}}=\left(\tilde{g}^{-1} \partial_{t} \tilde{g}\right)_{j} h^{i j}=\tilde{A}_{j} h^{i j}
$$

and inverting for the generalized velocities, one obtains the Hamiltonian:

$$
\tilde{H}=\frac{1}{2} \int_{\mathbb{R}} \mathrm{d} \sigma \tilde{I}^{i} \tilde{I}^{j} h_{i j}+\tilde{J}_{i} \tilde{J}_{j} h^{i j}=\frac{1}{2} \int_{\mathbb{R}} \mathrm{d} \sigma \tilde{I}_{I}\left(\tilde{\mathcal{K}}_{0}^{-1}\right)^{I J} \tilde{I}_{J}
$$

with

$$
\tilde{\mathcal{K}}_{0}=\left(\begin{array}{cc}
h^{i j} & 0 \\
0 & h_{i j}
\end{array}\right)
$$

and $\tilde{I}_{J}=\left(\tilde{I}^{j}, \tilde{J}_{j}\right)$, while the equal-time Poisson brackets can be derived in the usual way from the action functional (see appendix A) to be

$$
\begin{aligned}
& \left\{\tilde{I}^{i}(\sigma), \tilde{I}^{j}\left(\sigma^{\prime}\right)\right\}=f^{i j}{ }_{k} \tilde{I}^{k}(\sigma) \delta\left(\sigma-\sigma^{\prime}\right), \\
& \left\{\tilde{I}^{i}(\sigma), \tilde{J}_{j}\left(\sigma^{\prime}\right)\right\}=\tilde{J}_{k}(\sigma) f^{k i}{ }_{j} \delta\left(\sigma-\sigma^{\prime}\right)-\delta_{j}^{i} \delta^{\prime}\left(\sigma-\sigma^{\prime}\right), \\
& \left\{\tilde{J}_{i}(\sigma), \tilde{J}_{j}\left(\sigma^{\prime}\right)\right\}=0
\end{aligned}
$$

yielding the equations of motion

$$
\begin{aligned}
& \partial_{t} \tilde{I}^{i}(\sigma)=\tilde{I}^{s} \tilde{I}^{r} f^{j i}{ }_{s} h^{r j}-\tilde{J}_{r} \tilde{J}_{s} f^{s i}{ }_{j} h^{r j}+\delta_{j}^{i} h^{r j} \partial_{\sigma} \tilde{J}_{r} \\
& \partial_{t} \tilde{J}_{i}(\sigma)=\left(\tilde{I}^{s} \tilde{J}_{k} f^{k j}{ }_{i}+\delta_{i}^{j} \partial_{\sigma} \tilde{I}^{s}\right) h_{s j} .
\end{aligned}
$$


The Poisson brackets (4.35)-(4.37) realize the current algebra $\mathfrak{c}_{3}=\mathfrak{s} \mathfrak{b}(2, \mathbb{C})(\mathbb{R}) \ltimes \mathfrak{a}$, which we have already regarded as the limit $i \alpha \rightarrow 0$ of the algebra (3.34)-(3.36).

Similarly to the $\mathrm{SU}(2) \mathrm{PCM}$, the currents $(\tilde{J}, \tilde{I})$ may be identified with cotangent space left coordinates for $\mathrm{T}^{*} \mathrm{SB}(2, \mathbb{C})(\mathbb{R})$. However, differently from $\mathrm{T}^{*} \mathrm{SU}(2), \mathrm{T}^{*} \mathrm{SB}(2, \mathbb{C})$ is not symplectomorphic to $\operatorname{SL}(2, \mathbb{C})$, the two spaces being topologically different to start with. Therefore, certainly the model cannot be given an equivalent description in terms of an $\operatorname{SL}(2, \mathbb{C})(\mathbb{R})$ algebra. Indeed, it will be shown, in the next section, that the $\operatorname{SB}(2, \mathbb{C})$ PCM Hamiltonian obtained here through Legendre transform can be related to the DPCM models previously found, through a $B$-transformation, but not its Poisson algebra.

\subsubsection{Dual Born geometry}

Following the same approach as in section 3.1.2, let us recall that a left-invariant paraHermitian structure $(\tilde{\eta}, \tilde{\kappa})$ can be defined on $\mathrm{T}^{*} \mathrm{SB}(2, \mathbb{C})$, as discussed for $\mathrm{T}^{*} \mathrm{SU}(2)$, starting from its Lie algebra $\mathfrak{s} \mathfrak{b}(2, \mathbb{C}) \ltimes \mathbb{R}^{3}$. Thus $\tilde{\kappa}$ comes from the splitting of $\mathfrak{s} \mathfrak{b}(2, \mathbb{C}) \ltimes \mathbb{R}^{3}$ as a vector space and $\tilde{\eta}$ is obtained from the duality pairing. The fundamental two-form of such structure is denoted by $\tilde{\omega}$.

According to what has been done in section 3.1 .2 , let us start from the metric $\tilde{\mathcal{K}}_{0}$. It is easily verified that it is Riemannian, with determinant equal to 1 and such that

$$
\tilde{\mathcal{K}}_{0}^{T} \tilde{\eta} \tilde{\mathcal{K}}_{0}=\tilde{\eta}
$$

We consider the $\beta$-dependent $B$-transformation

$$
e^{B(\beta)}=\left(\begin{array}{cc}
\mathbb{1} & i \beta B \\
0 & \mathbb{1}
\end{array}\right) \in O(3,3)
$$

with $B^{i j}=\epsilon^{i j 3}$ as before and $\beta$ an imaginary parameter.

The Riemannian metric $\tilde{\mathcal{K}}_{\beta}$, can be obtained by the $B$-transformation acting on $\tilde{\mathcal{K}}_{0}$ :

$$
\tilde{\mathcal{K}}_{\beta}=\left(e^{-B(\beta)}\right)^{t} \tilde{\mathcal{K}}_{0} e^{B(\beta)},
$$

yielding

$$
\left(\tilde{\mathcal{K}}_{\beta}\right)_{I J}=\left(\begin{array}{cc}
h^{i j} & 2 i \beta \epsilon^{i l 3} \delta_{l j} \\
2 i \beta \delta_{i l} \epsilon^{j l 3} & \delta^{i j}-\epsilon_{i l 3} \delta^{l k} \epsilon_{j k 3}\left(2 \beta^{2}+\frac{1}{2}\right)
\end{array}\right) .
$$

Furthermore, the left-invariant para-Hermitian structure $(\tilde{\eta}, \tilde{\kappa})$ is transformed under $e^{B(\beta)}$ with

$$
\tilde{\kappa}_{\beta}=e^{B(\beta)} \tilde{\kappa} e^{-B(\beta)}
$$

still compatible with $\tilde{\eta}$, so that the fundamental two-form becomes $\tilde{\omega}_{\beta}=\tilde{\eta} \tilde{\kappa}_{\beta}$. In matrix form, the new almost para-Hermitian structure reads as:

$$
\kappa_{\beta}=\left(\begin{array}{cc}
\mathbb{1} & 2 i \beta B \\
0 & \mathbb{1}
\end{array}\right) \quad \eta=\left(\begin{array}{ll}
0 & \mathbb{1} \\
\mathbb{1} & 0
\end{array}\right) \quad \omega_{\beta}=\left(\begin{array}{cc}
0 & \mathbb{1} \\
\mathbb{1} & 2 i \beta \eta B
\end{array}\right)
$$


where $\eta B \in \Gamma\left(\wedge^{2} T^{*} \mathbb{R}^{3}\right)$. The new almost para-Hermitian structure still has $\operatorname{TSB}(2, \mathbb{C})$ as eigenbundle while $T \mathbb{R}^{3}$ is transformed in a non-involutive distribution $V_{\beta}$ whose sections are generated by vector fields in the form $\bar{Y}^{i}=Y^{i}+i \beta \epsilon^{i j 3} X_{j}$.

Let us compare these findings with the dual models constructed in the previous section. We find that the metric (4.43) is equal to $\mathcal{H}_{\tau, \alpha}{ }^{-1}$ in eq. (4.16), for the following values of the parameters

$$
\beta= \pm \frac{i}{2}, \quad \bar{\tau}= \pm i, \quad \alpha= \pm i .
$$

In terms of the new metric one thus obtains:

$$
\tilde{H}_{\beta}=\frac{1}{2} \int_{\mathbb{R}} \mathrm{d} \sigma \tilde{I}_{I}\left(\tilde{\mathcal{K}}_{\beta}^{-1}\right)^{I J} \tilde{I}_{J}
$$

which, for the choice of the parameters (4.46), reproduces the Hamiltonian (4.23) that we have obtained by duality from the PCM SU(2) model.

Notice however that, while the Poisson algebra of the dual models constructed in section 4 is the full affine algebra of $\mathfrak{s l}(2, \mathbb{C})$, here we only have a contraction of such an algebra, or in general a different algebra, after rotating the fields with the B-transformation (4.41).

Summarizing our findings, the natural $\mathrm{SB}(2, \mathbb{C})$ PCM model constructed in the Lagrangian approach, has an Hamiltonian formulation given by the Hamiltonian (4.33) and the Poisson algebra (4.35)-(4.37). On the other hand, the models which we have obtained in section 4 by performing a T-duality transformation of target space, namely an $O(3,3)$ rotation, are described by the Hamiltonians (4.23) and Poisson algebra (4.20)-(4.22). The relation between the two, if any, is still unclear to us.

\section{Double principal chiral model}

In the previous section we have succeeded in describing the Principal Chiral Modelof SU(2) in terms of currents whose Poisson brackets furnish a realization of the affine algebra of the group $\mathrm{SL}(2, \mathbb{C})$, hence exhibiting a larger symmetry than the original Lagrangian approach. Moreover, we have defined a natural model on the dual group of $\mathrm{SU}(2)$ and we have exhibited a transformation which relates the Riemannian metrics of the two models. It is therefore legitimate to look for a Lagrangian and an action with a manifest $\operatorname{SL}(2, \mathbb{C})$ symmetry.

\subsection{The Lagrangian formalism}

This is achieved by extending the $\operatorname{SL}(2, \mathbb{C})$ action for the Isotropic Rigid Rotor reviewed in section 2.2 to field theory. Hence, let us consider the group valued field:

$$
\Phi: \mathbb{R}^{1,1} \rightarrow \gamma \in \mathrm{SL}(2, \mathbb{C})
$$

and let us introduce the left-invariant Maurer-Cartan one-form $\gamma^{-1} \mathrm{~d} \gamma$ whose pull-back to $\mathbb{R}^{1,1}$ reads as:

$$
\Phi^{*}\left(\gamma^{-1} \mathrm{~d} \gamma\right)=\gamma^{-1} \partial_{t} \gamma \mathrm{d} t+\gamma^{-1} \partial_{\sigma} \gamma \mathrm{d} \sigma
$$


which takes values in the Lie algebra $\mathfrak{s l}(2, \mathbb{C})$. As previously, we shall not specify the pullback from now on, unless necessary. Hence, upon using the Lie algebra basis $e_{I}=\left(e_{i}, \tilde{e}^{i}\right)$ as in section 2.2 , one has:

$$
\begin{aligned}
\gamma^{-1} \partial_{t} \gamma & =\dot{\mathbf{Q}}^{I} e_{I}, \\
\gamma^{-1} \partial_{\sigma} \gamma & =\mathbf{Q}^{\prime I} e_{I},
\end{aligned}
$$

with $\dot{\mathbf{Q}}^{I}, \mathbf{Q}^{\prime I}$, left generalized coordinates, respectively given by:

$$
\dot{\mathbf{Q}}^{I}=\operatorname{Tr}\left(\gamma^{-1} \partial_{t} \gamma e_{I}\right), \quad \mathbf{Q}^{\prime I}=\operatorname{Tr}\left(\gamma^{-1} \partial_{\sigma} \gamma e_{I}\right)
$$

with $\operatorname{Tr}$ the Cartan-Killing metric of $\mathfrak{s l}(2, \mathbb{C})$. Moreover, as already done in eqs. (2.45), we can use the product (2.12) to project the fiber coordinates along the bialgebra summands $\mathfrak{s u}(2)$ and $\mathfrak{s} \mathfrak{b}(2, \mathbb{C})$, according to

$$
\begin{array}{rlrl}
\dot{Q}^{i}(\sigma, t) & =2 \operatorname{Im} \operatorname{Tr}\left(\gamma^{-1} \partial_{t} \gamma \tilde{e}^{i}\right) ; & \dot{\tilde{Q}}_{i}(\sigma, t)=2 \operatorname{Im} \operatorname{Tr}\left(\gamma^{-1} \partial_{t} \gamma e_{i}\right) \\
Q^{\prime i}(\sigma, t)=2 \operatorname{Im} \operatorname{Tr}\left(\gamma^{-1} \partial_{\sigma} \gamma \tilde{e}^{i}\right) ; & \tilde{Q}_{i}^{\prime}(\sigma, t)=2 \operatorname{Im} \operatorname{Tr}\left(\gamma^{-1} \partial_{\sigma} \gamma e_{i}\right) .
\end{array}
$$

The Hodge operator applied to the Maurer-Cartan one-form (5.1) exchanges the currents $\dot{\mathbf{Q}}^{I}$ and $\mathbf{Q}^{\prime I}$ so to give:

$$
*_{H} \Phi^{*}\left[\gamma^{-1} \mathrm{~d} \gamma\right]=\gamma^{-1} \partial_{t} \gamma \mathrm{d} \sigma+\gamma^{-1} \partial_{\sigma} \gamma \mathrm{d} t .
$$

We therefore postulate the following action functional:

$$
\mathcal{S}=\int_{\mathbb{R}^{2}} k_{1}\left\langle\Phi^{*}\left[\gamma^{-1} \mathrm{~d} \gamma\right] \wedge \underset{H}{*} \Phi^{*}\left[\gamma^{-1} \mathrm{~d} \gamma\right]\right\rangle+k_{2}\left(\left(\Phi^{*}\left[\gamma^{-1} \mathrm{~d} \gamma\right] \wedge \underset{H}{*} \Phi^{*}\left[\gamma^{-1} \mathrm{~d} \gamma\right]\right)\right)
$$

which is the natural extension to field theory of the action introduced for the rigid rotor in eq. (2.44). Upon introducing $k=k_{1} / k_{2}$, the Lagrangian is rewritten in terms of the left generalized coordinates

$$
\mathbf{L}=\frac{1}{2} \int_{\mathbb{R}} \mathrm{d} \sigma(k \eta+\mathcal{H})_{I J}\left(\dot{\mathbf{Q}}^{I} \dot{\mathbf{Q}}^{J}-\mathbf{Q}^{\prime I} \mathbf{Q}^{\prime J}\right)
$$

with

$$
(k \eta+\mathcal{H})_{I J}=\left(\begin{array}{cc}
\delta_{i j} & k \delta_{i}^{j}+\epsilon_{i}^{j 3} \\
k \delta_{j}^{i}-\epsilon_{j 3}^{i} & \left(\delta^{i j}+\epsilon_{k 3}^{i} \epsilon_{l 3}^{j} \delta^{k l}\right)
\end{array}\right) .
$$

Recall that $\eta$ (Lorentzian) and $\mathcal{H}$ (Riemannian) are the left-invariant metrics on $\operatorname{SL}(2, \mathbb{C})$ induced, respectively, by the pairings $2 \operatorname{Im} \operatorname{Tr}()$ and $2 \operatorname{Re} \operatorname{Tr}()$ on $\mathfrak{s l}(2, \mathbb{C})$. They are two of the structures defining a Born geometry on $\mathrm{SL}(2, \mathbb{C})$.

The Euler-Lagrange equation for the Lagrangian density (5.9) are:

$$
\partial_{t} \frac{\partial \mathbf{L}}{\partial \dot{\mathbf{Q}}^{J}}+\partial_{\sigma} \frac{\partial \mathbf{L}}{\partial \mathbf{Q}^{\prime J}}=(k \eta+\mathcal{H})_{I J}\left(\partial_{t} \dot{\mathbf{Q}}^{J}-\partial_{\sigma} \mathbf{Q}^{\prime J}\right)=\mathbf{L}_{\mathbf{X}_{J}} \mathbf{L}
$$

with $\mathbf{X}_{J}$ the left-invariant vector fields on the group manifold of $\operatorname{SL}(2, \mathbb{C})$. Before passing to the Hamiltonian description, let us stress that the generalized action describes a kind of non-linear sigma model with target space $\mathrm{SL}(2, \mathbb{C})$, hence with doubled dimension with respect to the previous models. Because the model only contains the currents $\dot{\mathbf{Q}}^{J}, \mathbf{Q}^{\prime J}$, as previously we can read the latter as the tangent space coordinates of $\operatorname{TSL}(2, \mathbb{C})(\mathbb{R})$. 


\subsection{The Hamiltonian formalism}

According to the remark made at the end of the previous subsection, the Hamiltonian model will be interpreted as a model over the cotangent space $T^{*} \operatorname{SL}(2, \mathbb{C})(\mathbb{R})$. In order to obtain the Hamiltonian of the system, the canonical momentum is computed:

$$
\mathbf{I}_{I}=\left(I_{i}, \tilde{I}^{i}\right)=\frac{\delta \mathbf{L}}{\delta \dot{\mathbf{Q}}^{I}}=(k \eta+\mathcal{H})_{I J} \dot{\mathbf{Q}}^{J} .
$$

Let us recall that the matrix $(k \eta+\mathcal{H})_{I J}$ is invertible for $k^{2} \neq 1$ and its inverse is

$$
\left[(k \eta+\mathcal{H})^{-1}\right]^{I J}=\frac{1}{2}\left(1-k^{2}\right)^{-1}\left(\begin{array}{cc}
\delta^{i j}+\epsilon_{l 3}^{i} \epsilon_{k 3}^{j} \delta^{l k} & -\epsilon^{i}{ }_{j 3}-k \delta_{j}^{i} \\
\epsilon_{i}{ }^{j 3}-k \delta_{i}^{j} & \delta_{i j}
\end{array}\right) .
$$

Therefore, the Legendre transform of (5.9), obtained by inverting (5.12), gives:

$$
\mathbf{H}=\frac{1}{2} \int_{\mathbb{R}} \mathrm{d} \sigma\left(\left[(k \eta+\mathcal{H})^{-1}\right]^{I J} \mathbf{I}_{I} \mathbf{I}_{J}+(k \eta+\mathcal{H})_{I J} \mathbf{J}^{I} \mathbf{J}^{J}\right),
$$

whereas we have for the Poisson brackets (see appendix A)

$$
\begin{aligned}
\left\{\mathbf{I}_{I}\left(\sigma^{\prime}\right), \mathbf{I}_{J}\left(\sigma^{\prime \prime}\right)\right\} & =C_{I J}{ }^{K} \mathbf{I}_{K} \delta\left(\sigma^{\prime}-\sigma^{\prime \prime}\right) \\
\left\{\mathbf{I}_{I}\left(\sigma^{\prime}\right), \mathbf{J}^{J}\left(\sigma^{\prime \prime}\right)\right\} & =C_{K I} \mathbf{J}^{K} \delta\left(\sigma^{\prime}-\sigma^{\prime \prime}\right)-\delta_{I}^{J} \delta^{\prime}\left(\sigma^{\prime}-\sigma^{\prime \prime}\right) \\
\left\{\mathbf{J}^{I}\left(\sigma^{\prime}\right), \mathbf{J}^{J}\left(\sigma^{\prime \prime}\right)\right\} & =0
\end{aligned}
$$

and we have renamed $\mathbf{Q}^{\prime I} \rightarrow \mathbf{J}^{I}$. The equations of motion read then as:

$$
\begin{aligned}
\dot{\mathbf{I}}_{J}= & \left.\left\{\mathbf{I}_{M}[k \eta+\mathcal{H})^{-1}\right]^{L K} \mathbf{I}_{K}-\mathbf{J}^{L}\left[(k \eta+\mathcal{H})^{-1}\right]_{L K} \mathbf{J}^{K}\right\} C_{J L}{ }^{M} \\
& +\partial_{\sigma} \mathbf{J}^{L}\left[(k \eta+\mathcal{H})^{-1}\right]_{L J}
\end{aligned}
$$

\subsection{Recovering the Chiral Model on TSU(2)}

Let us prove that we can recover the action on of the Principal Chiral Model on TSU(2) with an appropriate gauging of the global symmetries of the generalized action.

Let us recall that

$$
\Phi^{*}(\gamma \mathrm{d} \gamma)=\left(\gamma^{-1} \partial_{t} \gamma\right)^{I} e_{I} \mathrm{~d} t+\left(\gamma^{-1} \partial_{\sigma} \gamma\right)^{I} e_{I} \mathrm{~d} \sigma
$$

can be projected along the two Lie algebras according to

$$
\begin{aligned}
\gamma^{-1} \partial_{t} \gamma & =A^{i} e_{i}+\tilde{A}_{i} \tilde{e}^{i} \\
\gamma^{-1} \partial_{\sigma} \gamma & =B^{i} e_{i}+\tilde{B}_{i} \tilde{e}^{i}
\end{aligned}
$$

with

$$
\begin{array}{ll}
A^{i}=2 \operatorname{Im} \operatorname{Tr} \gamma^{-1} \partial_{t} \gamma \tilde{e}^{i} & \tilde{A}_{i}=2 \operatorname{Im} \operatorname{Tr} \gamma^{-1} \partial_{t} \gamma t e_{i} \\
B^{i}=2 \operatorname{Im} \operatorname{Tr} \gamma^{-1} \partial_{\sigma} \gamma \tilde{e}^{i} & \tilde{B}_{i}=2 \operatorname{Im} \operatorname{Tr} \gamma^{-1} \partial_{\sigma} \gamma t e_{i}
\end{array}
$$


We notice that, fixing the decomposition $\gamma=\tilde{g} g$, with $\tilde{g} \in \mathrm{SB}(2, \mathbb{C})$ and $g \in \mathrm{SU}(2)$, for any element $\gamma \in \mathrm{SL}(2, \mathbb{C})$, the action (5.8) has manifest global symmetry under left action of $\mathrm{SB}(2, \mathbb{C})$, called $\mathrm{SB}(2, \mathbb{C})_{L}$, and $\mathrm{SU}(2)_{R}$, the right action of $\mathrm{SU}(2)$. We let the $\mathrm{SB}(2, \mathbb{C})_{L}$ symmetry become local, so we can introduce the connection one-form $C=C_{i} \tilde{e}^{i}$ on the principal bundle $\mathbb{R}^{1,1} \times \mathrm{SB}(2, \mathbb{C}) \rightarrow \mathbb{R}^{1,1}$ so that its pull-back (along any section) to $\mathbb{R}^{1,1}$ reads $C_{i}^{t} \tilde{e}^{i} \mathrm{~d} t+C_{i}^{\sigma} \tilde{e}^{i} \mathrm{~d} \sigma$, which takes values in the Lie algebra $\mathfrak{s} \mathfrak{b}(2, \mathbb{C})$. Hence we modify the left-invariant one-form with the covariant derivative $\mathrm{D}=\mathrm{d}+C$ :

$$
\Phi^{*}\left(\gamma^{-1} \mathrm{D} \gamma\right)=\Phi^{*}\left(\gamma^{-1} \mathrm{~d} \gamma\right)+\Phi^{*}\left(\gamma^{-1} C \gamma\right)=\left(\gamma^{-1} \partial_{t} \gamma+\gamma^{-1} C^{t} \gamma\right) \mathrm{d} t+\left(\gamma^{-1} \partial_{\sigma} \gamma+\gamma^{-1} C^{\sigma} \gamma\right) \mathrm{d} t
$$

and define

$$
\gamma^{-1} \partial_{t} \gamma+\gamma^{-1} C^{t} \gamma=U_{i} \tilde{e}^{i}+W^{i} e_{i}
$$

where

$$
\begin{aligned}
U_{i} & =A_{i}+C_{j}^{t} 2 \operatorname{Im} \operatorname{Tr}\left(\gamma^{-1} \tilde{e}^{j} \gamma e_{i}\right) \\
W^{i} & =A^{i}+C_{j}^{t} 2 \operatorname{Im} \operatorname{Tr}\left(\gamma^{-1} \tilde{e}^{j} \gamma \tilde{e}^{i}\right)
\end{aligned}
$$

and similarly

$$
\gamma^{-1} \partial_{\sigma} \gamma+\gamma^{-1} C^{\sigma} \gamma=V^{i} e_{i}+Z_{i} \tilde{e}^{i}
$$

with

$$
\begin{aligned}
V^{i} & =B^{i}+C_{j}^{\sigma} 2 \operatorname{Im} \operatorname{Tr}\left(\gamma^{-1} \tilde{e}^{j} \gamma \tilde{e}^{i}\right) \\
Z_{i} & =B_{i}+C_{j}^{\sigma} 2 \operatorname{Im} \operatorname{Tr}\left(\gamma^{-1} \tilde{e}^{j} \gamma e_{i}\right) .
\end{aligned}
$$

In terms of the new degrees of freedom the generalized action (5.8), with the gauge connection added, reads as:

$$
S_{C}=\frac{1}{2} \int_{\mathbb{R}^{2}}\left[\delta_{i j} W^{i} W^{j}+2\left(k \delta_{i}^{j}+\epsilon_{i}^{j 3}\right) W^{i} U_{j}+h^{i j} U_{i} U_{j}-\delta_{i j} V^{i} V^{j}+2\left(k \delta_{i}^{j}+\epsilon_{i}^{j 3}\right) V^{i} Z_{j}+h^{i j} Z_{i} Z_{j}\right]
$$

On performing the following transformations

$$
\begin{aligned}
\hat{W}^{i} & =W^{i}+\left(k \delta^{i j}-\epsilon^{i s 3}\right) U_{s} \\
\hat{V}^{i} & =V^{i}+\left(k \delta^{i j}-\epsilon^{i s 3}\right) Z_{s}
\end{aligned}
$$

while $U_{i}, Z_{i}$ remaining unchanged, one gets for $S_{C}$ the following expression:

$$
S_{C}=\frac{1}{2} \int_{\mathbb{R}^{2}}\left[\delta_{i j}\left(\hat{W}^{i} \hat{W}^{j}-\hat{V}^{i} \hat{V}^{j}\right)+\left(1-k^{2}\right) \delta^{i j}\left(U_{i} U_{j}-Z_{i} Z_{j}\right)\right] \mathrm{d} \sigma \mathrm{d} t
$$

The Wick-rotated generating functional of the gauged theory reads then as:

$$
Z_{C}=\int \mathcal{D} g \mathcal{D} \tilde{g} \mathcal{D} C^{t} \mathcal{D} C^{\sigma} e^{-S^{E}{ }_{C}}
$$


with $S_{C}^{E}$ the Euclidean gauge action, and we can trade the integration over $C^{t}, C^{\sigma}$ by an integration over the fields $U_{i}, Z_{i}$

$$
\begin{aligned}
Z_{C}= & \int \mathcal{D} g \mathcal{D} \tilde{g} \operatorname{det}\left(\frac{\delta C_{i}^{t}}{\delta U_{j}}\right) \operatorname{det}\left(\frac{\delta C_{i}^{\sigma}}{\delta Z_{j}}\right) e^{-\frac{1}{2} \int_{\mathbb{R}^{2}} \mathrm{~d} t \mathrm{~d} \sigma \delta_{i j}\left(\hat{W}^{i} \hat{W}^{j}+\hat{V}^{i} \hat{V}^{j}\right)} \\
& \times \int \mathcal{D} U_{i} e^{-\frac{1}{2} \int_{\mathbb{R}^{2}} \mathrm{~d} t \mathrm{~d} \sigma\left(1-k^{2}\right) \delta^{i j} U_{i} U_{j}} \int \mathcal{D} Z_{i} e^{-\frac{1}{2} \int_{\mathbb{R}^{2}} \mathrm{~d} t \mathrm{~d} \sigma\left(1-k^{2}\right) \delta^{i j} Z_{i} Z_{j}} .
\end{aligned}
$$

For $-1 \leq k \leq 1$ the last two functional integrals can be performed yielding:

$$
\int \mathcal{D} U_{i} e^{-\frac{1}{2} \int_{\mathbb{R}^{2}} \mathrm{~d} t \mathrm{~d} \sigma\left(1-k^{2}\right) \delta^{i j} U_{i} U_{j}}=\int \mathcal{D} Z_{i} e^{-\frac{1}{2} \int_{\mathbb{R}^{2}} \mathrm{~d} t \mathrm{~d} \sigma\left(1-k^{2}\right) \delta^{i j} Z_{i} Z_{j}}=\left(\frac{2 \pi}{1-k^{2}}\right)^{\frac{3}{2}}
$$

Similarly, the Jacobian determinants appearing in (5.35) are constant, because the gauge transformation only involves constant matrices (see [79] for details). Therefore, up to a regularization factor which has to be introduced to take care of the volume integration over the group $\mathrm{SB}(2, \mathbb{C})$, we are left with

$$
Z=\int \mathcal{D} g e^{-\frac{1}{2} \int_{\mathbb{R}^{2}} \mathrm{~d} t \mathrm{~d} \sigma \delta_{i j}\left(\hat{W}^{i} \hat{W}^{j}+\hat{V}^{i} \hat{V}^{j}\right)}
$$

Upon observing that the transformations (5.31), (5.32) give a redefinition of the fields $W^{i}$, $V^{i}$ as $\hat{W}^{i}, \hat{V}^{i}$ still $\mathfrak{s u}(2)$-valued, the partition function (5.37) clearly involves the Action of the Principal Chiral Model on the group SU(2). Indeed, we can write the exponent of the derived partition function as

$$
S=\frac{1}{2} \int_{\mathbb{R}^{2}} \operatorname{Tr}\left(g^{\prime-1} \mathrm{~d} g^{\prime} \wedge * g^{\prime-1} \mathrm{~d} g^{\prime}\right),
$$

with $g^{\prime} \in \mathrm{SU}(2)$, so we explicitly have the derivation of the model on $\mathrm{SU}(2)$. Gauging the other symmetry, we obtain the model on $\mathrm{SB}(2, \mathbb{C})$ as we discussed for the Isotropic Rigid Rotor, see [79] for details.

\section{Conclusions and outlook}

An alternative parametrization of the SU(2) Principal Chiral Model found in refs. [15, 16], shows that the PCM, in its Hamiltonian formulation, can be given an equivalent description in terms of currents which span a target phase space isomorphic to the group manifold of $\mathrm{SL}(2, \mathbb{C})$. Their Poisson algebra can be given the structure of the centrally extended affine algebra $\mathfrak{s l}(2, \mathbb{C})(\mathbb{R})$. Following a previous paper of the authors, [79], the model is here studied as a higher dimensional generalization of the Isotropic Rigid Rotor dynamics with the aim of further deepening its remarkable geometric structures.

The standard Hamiltonian formulation of the SU(2) PCM model exploits the fact that the dynamics is fully described by fields, the currents, which span $\mathrm{T}^{*} \mathrm{SU}(2)$ as target phase space and act as infinitesimal generators of an affine algebra which is the semidirect sum $\mathfrak{s u}(2)(\mathbb{R}) \dot{\oplus} \mathfrak{a}(\mathbb{R})$. We speculate on the fact that, as a Lie group, $\mathrm{T}^{*} \mathrm{SU}(2)$ is the trivial Drinfel'd double of the group SU(2), which we have called the classical double. 
The latter gives rise to a fully nontrivial Drinfel'd double, the group $\operatorname{SL}(2, \mathbb{C})$, when the Abelian subalgebra of the semidirect sum is deformed to that of $\operatorname{SB}(2, \mathbb{C})$. By exploiting this property, we first review in detail the derivation of a whole family of equivalent PCM models described in terms of current algebra of the group $\mathrm{SL}(2, \mathbb{C})$, we thus show that they can actually be interpreted in terms of Born geometries related by B-transformations. We then perform $O(3,3)$ transformations of such a family and find a parametric family of T-dual PCM models, with target configuration space the group $\operatorname{SB}(2, \mathbb{C})$, the Poisson-Lie dual of $\mathrm{SU}(2)$ in the Iwasawa decomposition of the Drinfel'd double $\mathrm{SL}(2, \mathbb{C})$. Poisson-Lie symmetries are discussed. Then, a natural Lagrangian model has been constructed directly on the dual group $\mathrm{SB}(2, \mathbb{C})$. Its relation to the dual models previously introduced is still unclear to us and needs further analysis. Finally we have introduced a double PCM with the group manifold of $\operatorname{SL}(2, \mathbb{C})$ as its target configuration space and $\operatorname{TSL}(2, \mathbb{C})$ as the target tangent space. The degrees of freedom are thus doubled. We have shown, performing a gauging of its symmetries, that both the Lagrangian models, with $\mathrm{SU}(2)$ and $\mathrm{SB}(2, \mathbb{C})$ target configuration spaces, can be retrieved.

A further extension of this model can be given adding a Wess-Zumino term [97]. This could provide a deeper insight, among other things, on the geometric structures of String Theory on $A d S_{3}$, the study of which is interesting from the point of view of the $A d S / C F T$ correspondence since it enables to study the correspondence beyond the gravity approximation [98-100].

Last but not least, all what we have learnt from this model could be further extended to the world-sheet string action. In this case, a manifestly $O(d, d)$-invariant action may be written, considering that the configuration space is no longer a Lie group, but a differentiable manifold. It would be interesting to follow this way, in which $O(d, d)$-invariance is implemented writing a doubled string action, as discussed for Principal Chiral Models, and then performing the low energy limit. This limit result should reproduce all the results so far obtained in Double Field Theory.

\section{Acknowledgments}

P.V. acknowledges support by COST (European Cooperation in Science and Technology) in the framework of COST Action MP1405 QSPACE. V.E.M. thanks Richard Szabo for helpful discussions. The work of V.E.M. was funded by the Doctoral Training Grant ST/R504774/1 from the U.K. Science and Technology Facilities Council (STFC). F.P. thanks the Simon Center for Geometry and Physics for their hospitality and support during the Simons Summer Workshop 2018.

\section{A Poisson brackets}

In this appendix we derive the current algebras (3.14)-(3.16), (4.35)-(4.37), (5.14)-(5.16) from the canonical one-form obtained by the relevant action functional. 
Let us start with the standard formulation of the principal $\mathrm{SU}(2)$ chiral model, whose action is given by (3.3). As for the rigid rotor, we choose the parametrization

$$
\phi: \sigma \in \mathbb{R} \rightarrow g(\sigma)=2\left(y^{0}(\sigma) e_{0}+i y^{i}(\sigma) e_{i}\right)
$$

with $\sum_{\mu} y^{\mu} y^{\mu}=1$ Upon defining $I=-\frac{i}{2} I_{i} e^{i *}$ with $e^{i *}\left(e_{j}\right)=\delta_{j}^{i}$, and recalling that $g^{-1} \mathrm{~d} g=$ $2 i \alpha^{k} e_{k}$, we have for the canonical one-form

$$
\Theta=\int_{\mathbb{R}}<I \mid g^{-1} \mathrm{~d} g>=\int_{\mathbb{R}} I_{i}(\sigma) \alpha^{i}(\sigma)
$$

so that

$$
\Omega=\int_{\mathbb{R}} \mathrm{d} I_{i}(\sigma) \wedge \alpha^{i}(\sigma)+I_{i}(\sigma) \epsilon_{j k}^{i} \alpha^{j}(\sigma) \wedge \alpha^{k}(\sigma)
$$

with $\alpha^{i}(\sigma)=\left[y^{0} \mathrm{~d} y^{i}-y^{i} \mathrm{~d} y^{0}+\epsilon_{j k}{ }^{i} y^{j} \mathrm{~d} y^{k}\right](\sigma)$ the left-invariant one-forms on the group manifold, in the chosen parametrization. The Poisson structure is thus

$$
\Lambda=\int_{\mathbb{R}} \mathrm{d} \sigma\left(X_{i}(\sigma) \wedge \frac{\delta}{\delta I_{i}(\sigma)}+\epsilon_{j k}^{i} I_{i} \frac{\delta}{\delta I_{j}(\sigma)} \wedge \frac{\delta}{\delta I_{k}(\sigma)}\right)
$$

with $X_{i}(\sigma)$ the left-invariant vector fields which are dual to the one-forms $\alpha^{i}(\sigma)$, that is, in the chosen parametrization

$$
X_{i}(\sigma)=y^{0} \frac{\delta}{\delta y^{i}(\sigma)}-y^{i} \frac{\delta}{\delta y^{0}(\sigma)}+\epsilon_{i j}^{k} y^{j}(\sigma) \frac{\delta}{\delta y^{k}(\sigma)}
$$

We thus obtain

$$
\begin{aligned}
\left\{I_{i}\left(\sigma^{\prime}\right), I_{j} \sigma^{\prime \prime}\right\} & =\epsilon_{i j}{ }^{k} I_{k}\left(\sigma^{\prime}\right) \delta\left(\sigma^{\prime}-\sigma^{\prime \prime}\right) \\
\left\{y^{i}\left(\sigma^{\prime}\right), I_{j}\left(\sigma^{\prime \prime}\right)\right\} & =\left[\delta_{j}^{i} y^{0}\left(\sigma^{\prime}\right)+\epsilon_{j k}{ }^{i} y^{k}\left(\sigma^{\prime}\right)\right] \delta\left(\sigma^{\prime}-\sigma^{\prime \prime}\right) \text { or }\left\{g\left(\sigma^{\prime}\right), I_{j}\left(\sigma^{\prime \prime}\right)\right\}=2 i g\left(\sigma^{\prime}\right) e_{j} \delta\left(\sigma^{\prime}-\sigma^{\prime \prime}\right) \\
\left\{y^{0}\left(\sigma^{\prime}\right), I_{j}\left(\sigma^{\prime \prime}\right)\right\} & =-y^{j}\left(\sigma^{\prime}\right) \delta\left(\sigma^{\prime}-\sigma^{\prime \prime}\right) \\
\left\{y^{\mu}\left(\sigma^{\prime}\right), y^{\nu}\left(\sigma^{\prime \prime}\right)\right\} & =0 \quad \text { or }\left\{g\left(\sigma^{\prime}\right), g\left(\sigma^{\prime \prime}\right)\right\}=0
\end{aligned}
$$

On using $J^{i}(\sigma)=-i \operatorname{Tr}\left(g^{-1} \partial_{\sigma} g\right) e_{i}=y^{0} \partial_{\sigma} y^{i}-y^{i} \partial_{\sigma} y^{0}+\epsilon_{j k}{ }^{i} y^{j} \partial_{\sigma} y^{k}$ we compute

$$
\begin{aligned}
\left\{J^{i}\left(\sigma^{\prime}\right), I_{j}\left(\sigma^{\prime \prime}\right)\right\} & =\operatorname{Tr} e_{i}\left\{g^{-1} \partial_{\sigma^{\prime}} g, I_{j}\left(\sigma^{\prime \prime}\right)\right\} \\
& =-i \operatorname{Tr} e_{i}\left[-g^{-1}\left\{g\left(\sigma^{\prime}\right), I_{j}\left(\sigma^{\prime \prime}\right)\right\} g^{-1} \partial_{\sigma^{\prime}} g+g^{-1}\left\{\partial_{\sigma^{\prime}} g, I_{j}\left(\sigma^{\prime \prime}\right)\right\}\right]
\end{aligned}
$$

which can be seen to give (3.15) because of the second of the brackets (A.6). Analogously we can compute

$$
\left\{J^{i}\left(\sigma^{\prime}\right), J^{j}\left(\sigma^{\prime \prime}\right)\right\}=\left\{\operatorname{Tr} e_{i} g^{-1} \partial_{\sigma^{\prime}} g, \operatorname{Tr} e_{j} g^{-1} \partial_{\sigma^{\prime \prime}} g\right\}=0
$$

because group variables have zero Poisson brackets according to the last of eqs. (A.6).

An analogous computation can be performed for the Poisson brackets of the chiral model on the Poisson-Lie dual group $\operatorname{SB}(2, \mathbb{C})$. The action functional for the model is represented by (4.25). As in section 2.1 we choose the parametrization

$$
\tilde{\phi}: \sigma \in \mathbb{R} \rightarrow \tilde{g}(\sigma)=2\left(u^{0}(\sigma) \tilde{e}^{0}+i u^{i}(\sigma) \tilde{e}^{i}\right)
$$


with $\left(u^{0}\right)^{2}-\left(u^{3}\right)^{2}=1$. On introducing $\tilde{I}=-i \tilde{I}^{i} \tilde{e}_{i}^{*}$, with $\tilde{e}_{i}^{*}\left(\tilde{e}^{j}\right)=\delta_{i}^{j}$ and recalling that $\tilde{g}^{-1} \mathrm{~d} \tilde{g}=i \tilde{\alpha}_{j} \tilde{e}^{j}$ We have for the canonical one-form

$$
\tilde{\Theta}=\int_{\mathbb{R}}<\tilde{I} \mid \tilde{g}^{-1} \mathrm{~d} \tilde{g}>=\int_{\mathbb{R}} \tilde{I}^{i}(\sigma) \tilde{\alpha}_{i}
$$

so that

$$
\tilde{\Omega}=\int_{\mathbb{R}} \mathrm{d} \tilde{I}^{i}(\sigma) \wedge \tilde{\alpha}_{i}(\sigma)+\tilde{I}^{i}(\sigma) f^{j k}{ }_{i} \tilde{\alpha}_{j}(\sigma) \wedge \tilde{\alpha}_{k}(\sigma)
$$

with $\tilde{\alpha}_{i}(\sigma)=2\left[u^{0} \mathrm{~d} u^{i}-u^{i} \mathrm{~d} u^{0}+f^{j k}{ }_{i} u_{j} \mathrm{~d} u_{k}\right](\sigma)$ the left-invariant one-forms on the group manifold, in the chosen parametrization. The Poisson structure is thus

$$
\tilde{\Lambda}=\int_{\mathbb{R}} \tilde{X}^{i}(\sigma) \wedge \frac{\delta}{\delta \tilde{I}^{i}(\sigma)}+f^{j k}{ }_{i} \tilde{I}^{i} \frac{\delta}{\delta \tilde{I}^{j}(\sigma)} \wedge \frac{\delta}{\delta \tilde{I}^{k}(\sigma)}
$$

with $\tilde{X}^{i}(\sigma)$ the left-invariant vector fields which are dual to the one-forms, that is, in the chosen parametrization

$$
\tilde{X}^{i}(\sigma)=\frac{1}{2}\left(u^{0} \frac{\delta}{\delta u^{i}(\sigma)}-u^{i} \frac{\delta}{\delta u^{0}(\sigma)}-f_{j}^{i k} u^{j}(\sigma) \frac{\delta}{\delta u^{k}(\sigma)}\right)
$$

We thus obtain

$$
\begin{aligned}
\left\{\tilde{I}^{i}\left(\sigma^{\prime}\right), \tilde{I}^{j} \sigma^{\prime \prime}\right\} & =f_{k}^{i j} \tilde{I}^{k}\left(\sigma^{\prime}\right) \delta\left(\sigma^{\prime}-\sigma^{\prime \prime}\right) \\
\left\{u^{i}\left(\sigma^{\prime}\right), \tilde{I}^{j}\left(\sigma^{\prime \prime}\right)\right\} & =\frac{1}{2}\left[\delta^{i j} u^{0}\left(\sigma^{\prime}\right)+f_{k}^{i j} u^{k}\left(\sigma^{\prime}\right)\right] \delta\left(\sigma^{\prime}-\sigma^{\prime \prime}\right) \text { or }\left\{\tilde{g}\left(\sigma^{\prime}\right), \tilde{I}^{j}\left(\sigma^{\prime \prime}\right)\right\}=2 \tilde{g}\left(\sigma^{\prime}\right) \tilde{e}^{j} \delta\left(\sigma^{\prime}-\sigma^{\prime \prime}\right) \\
\left\{u^{0}\left(\sigma^{\prime}\right), \tilde{I}^{j}\left(\sigma^{\prime \prime}\right)\right\} & =-\frac{1}{2} u^{j}\left(\sigma^{\prime}\right) \delta\left(\sigma^{\prime}-\sigma^{\prime \prime}\right) \\
\left\{u^{\mu}\left(\sigma^{\prime}\right), u^{\nu}\left(\sigma^{\prime \prime}\right)\right\} & =0 \quad \text { or }\left\{\tilde{g}\left(\sigma^{\prime}\right), \tilde{g}\left(\sigma^{\prime \prime}\right)\right\}=0
\end{aligned}
$$

On using $\tilde{J}_{i}(\sigma)=\left\langle\tilde{g}^{-1} \partial_{\sigma} \tilde{g}, e_{i}\right\rangle=u^{0} \partial_{\sigma} u^{i}-u^{i} \partial_{\sigma} u^{0}+f^{i k}{ }_{j} u^{j} \partial_{\sigma} u^{k}$ we compute

$$
\begin{aligned}
\left\{\tilde{J}_{i}\left(\sigma^{\prime}\right), \tilde{I}^{j} \sigma^{\prime \prime}\right\} & =2 \operatorname{Im} \operatorname{Tr} e_{i}\left\{\tilde{g}^{-1} \partial_{\sigma^{\prime}} \tilde{g}, \tilde{I}^{j}\left(\sigma^{\prime \prime}\right)\right\} \\
& =2 \operatorname{Im} \operatorname{Tr} e_{i}\left[-\tilde{g}^{-1}\left(\sigma^{\prime}\right)\left\{\tilde{g}\left(\sigma^{\prime}\right), \tilde{I}^{j}\left(\sigma^{\prime \prime}\right\} \tilde{g}^{-1}\left(\sigma^{\prime}\right) \partial_{\sigma^{\prime}} \tilde{g}+\tilde{g}^{-1}\left\{\partial_{\sigma^{\prime}} \tilde{g}, \tilde{I}^{j}\right\}\right]\right.
\end{aligned}
$$

which can be seen to give (4.36) because of the second of the brackets (A.14). Similarly we can compute

$$
\left\{\tilde{J}^{i}\left(\sigma^{\prime}\right), \tilde{J}^{j} \sigma^{\prime \prime}\right\}=\left\{2 \operatorname{Im} \operatorname{Tr} e_{i} \tilde{g}^{-1} \partial_{\sigma^{\prime}} \tilde{g}, 2 \operatorname{Im} \operatorname{Tr} e_{j} \tilde{g}^{-1} \partial_{\sigma^{\prime \prime}} \tilde{g}\right\}
$$

where the latter is zero because group variables have zero Poisson brackets according to last of eqs. (A.14).

Finally, we derive the Poisson brackets $(5.14)-(5.16)$ for the $\mathfrak{s l}(2, \mathbb{C})(\mathbb{R})$ current algebra. Upon defining $\mathbf{I}=-\frac{1}{2} \mathbf{I}_{I} e^{I^{*}}$ with $e^{I^{*}}\left(e_{I}\right)=\delta_{J}^{I}$, and recalling that $\gamma^{-1} \mathrm{~d} \gamma=2 \zeta^{K} e_{K}$, with $\zeta^{K}$ the $\mathrm{SL}(2, \mathbb{C})$ left-invariant one-forms, we have for the canonical one-form

$$
\Theta_{D}=\int_{\mathbb{R}}<\mathbf{I} \mid \gamma^{-1} \mathrm{~d} \gamma>=\int_{\mathbb{R}} \mathbf{I}_{I}(\sigma) \zeta^{I}(\sigma)
$$


so that

$$
\Omega_{D}=\int_{\mathbb{R}} \mathrm{d} \mathbf{I}_{I}(\sigma) \wedge \zeta^{I}(\sigma)+\mathbf{I}_{I}(\sigma) C_{J K}^{I} \zeta^{J}(\sigma) \wedge \zeta^{K}(\sigma)
$$

The Poisson structure is thus

$$
\Lambda_{D}=\int_{\mathbb{R}} \mathrm{d} \sigma\left(\mathbf{X}_{I}(\sigma) \wedge \frac{\delta}{\delta \mathbf{I}_{I}(\sigma)}+C_{J K}{ }^{I} \mathbf{I}_{I}(\sigma) \frac{\delta}{\delta \mathbf{I}_{J}(\sigma)} \wedge \frac{\delta}{\delta \mathbf{I}_{K}(\sigma)}\right)
$$

with $\mathbf{X}_{I}(\sigma)$ the left-invariant vector fields which are dual to the one-forms $\zeta^{I}(\sigma)$.

We thus compute the Poisson brackets. For the sake of simplicity, we do not choose any parametrization for $\operatorname{SL}(2, \mathbb{C})$. A similar computation can be analogously carried on for the Poisson brackets on $\mathrm{SU}(2)$ and $\mathrm{SB}(2, \mathbb{C})$, since we always deal with matrix Lie groups. The first Poisson bracket is straightforward

$$
\left\{\mathbf{I}_{I}\left(\sigma^{\prime}\right), \mathbf{I}_{J}\left(\sigma^{\prime \prime}\right)\right\}=\Lambda_{D}\left(\mathrm{~d} \mathbf{I}_{I}\left(\sigma^{\prime}\right), \mathrm{d} \mathbf{I}_{J}\left(\sigma^{\prime \prime}\right)\right)=C_{I J}{ }^{K} \mathbf{I}_{K}\left(\sigma^{\prime}\right) \delta\left(\sigma^{\prime}-\sigma^{\prime \prime}\right) .
$$

In order to derive the remaining brackets, we compute

$$
\begin{aligned}
\left\{\gamma\left(\sigma^{\prime}\right), \mathbf{I}_{J}\left(\sigma^{\prime \prime}\right)\right\} & =\Lambda_{D}\left(\mathrm{~d} \gamma\left(\sigma^{\prime}\right), \mathrm{d} \mathbf{I}_{J}\left(\sigma^{\prime \prime}\right)\right) \\
& =\mathbf{X}_{J}\left(\sigma^{\prime \prime}\right)\left(\gamma\left(\sigma^{\prime}\right) \gamma^{-1}\left(\sigma^{\prime}\right) \mathrm{d} \gamma\left(\sigma^{\prime}\right)\right) \\
& =2 \gamma\left(\sigma^{\prime}\right) e_{J} \delta\left(\sigma^{\prime}-\sigma^{\prime \prime}\right) .
\end{aligned}
$$

Notice that we could have performed the same calculation for the groups $\mathrm{SU}(2)$ and $\mathrm{SB}(2, \mathbb{C})$ where the analogous result was instead obtained by choosing explicitly a parametrization. The above calculation can be carried on for any matrix Lie group. Finally,

$$
\left\{\gamma\left(\sigma^{\prime}\right), \gamma\left(\sigma^{\prime \prime}\right)\right\}=0
$$

because there are no terms in $\Lambda_{D}$ involving the wedge product of two left-invariant vector fields. On using $\mathbf{J}^{I}(\sigma)=\operatorname{Tr}\left(\gamma^{-1} \partial_{\sigma} \gamma\right) e_{I}$, we compute

$$
\begin{aligned}
\left\{\mathbf{J}^{I}\left(\sigma^{\prime}\right), \mathbf{I}_{J}\left(\sigma^{\prime \prime}\right)\right\} & =\operatorname{Tr} e_{I}\left\{\gamma^{-1} \partial_{\sigma^{\prime}} \gamma, \mathbf{I}_{J}\left(\sigma^{\prime \prime}\right)\right\} \\
& =\operatorname{Tr} e_{I}\left[-\gamma^{-1}\left\{\gamma\left(\sigma^{\prime}\right), \mathbf{I}_{J}\left(\sigma^{\prime \prime}\right)\right\} \gamma^{-1} \partial_{\sigma^{\prime}} \gamma+\gamma^{-1}\left\{\partial_{\sigma^{\prime}} \gamma, \mathbf{I}_{J}\left(\sigma^{\prime \prime}\right)\right\}\right]
\end{aligned}
$$

which can be seen to give (5.15) because of the Poisson brackets (A.21). Analogously we can compute

$$
\left\{\mathbf{J}^{I}\left(\sigma^{\prime}\right), \mathbf{J}^{J}\left(\sigma^{\prime \prime}\right)\right\}=\left\{\operatorname{Tr} e_{I} \gamma^{-1} \partial_{\sigma^{\prime}} \gamma, \operatorname{Tr} e_{J} \gamma^{-1} \partial_{\sigma^{\prime \prime}} \gamma\right\}=0,
$$

which gives (5.16) because group variables have zero Poisson brackets according to eq. (A.22).

Open Access. This article is distributed under the terms of the Creative Commons Attribution License (CC-BY 4.0), which permits any use, distribution and reproduction in any medium, provided the original author(s) and source are credited. 


\section{References}

[1] A. Giveon, M. Porrati and E. Rabinovici, Target space duality in string theory, Phys. Rept. 244 (1994) 77 [hep-th/9401139] [INSPIRE].

[2] E. Alvarez, L. Álvarez-Gaumé and Y. Lozano, An Introduction to T duality in string theory, Nucl. Phys. Proc. Suppl. 41 (1995) 1 [hep-th/9410237] [InSPIRE].

[3] M.J. Duff, Duality Rotations in String Theory, Nucl. Phys. B 335 (1990) 610 [INSPIRE].

[4] T.H. Buscher, A Symmetry of the String Background Field Equations, Phys. Lett. B 194 (1987) 59 [INSPIRE].

[5] T.H. Buscher, Path Integral Derivation of Quantum Duality in Nonlinear $\sigma$-models, Phys. Lett. B 201 (1988) 466 [INSPIRE].

[6] M. Roček and E.P. Verlinde, Duality, quotients and currents, Nucl. Phys. B 373 (1992) 630 [hep-th/9110053] [INSPIRE].

[7] X.C. de la Ossa and F. Quevedo, Duality symmetries from non-Abelian isometries in string theory, Nucl. Phys. B 403 (1993) 377 [hep-th/9210021] [InSPIRE].

[8] C. Klimčík and P. Ševera, Dual non-Abelian duality and the Drinfeld double, Phys. Lett. B 351 (1995) 455 [hep-th/9502122] [INSPIRE].

[9] C. Klimčík and P. Ševera, Poisson-Lie T duality and loop groups of Drinfeld doubles, Phys. Lett. B 372 (1996) 65 [hep-th/9512040] [INSPIRE].

[10] C. Klimčík, Poisson-Lie T duality, Nucl. Phys. Proc. Suppl. 46 (1996) 116 [hep-th/9509095] [INSPIRE].

[11] V.G. Drinfeld, Hamiltonian Lie groups, Lie bialgebras and the geometric meaning of the classical Yang-Baxter equation, Sov. Math. Dokl. 27 (1983) 68.

[12] V.G. Drinfeld, Quantum Groups, Proceedings of the International Congress of Mathematicians, Berkeley, U.S.A., 1986, American Mathematical Society, Providence U.S.A. (1987), pp. 798-820.

[13] M.A. Semenov-Tian-Shansky, Poisson Lie groups, quantum duality principle and the quantum double, Theor. Math. Phys. 93 (1992) 1292 [hep-th/9304042] [INSPIRE].

[14] Y. Kossmann-Schwarzbach, Lie bialgebras, Poisson Lie groups and dressing transformations, in Integrability of Nonlinear Systems, Second edition, Lecture Notes in Physics 638, Springer-Verlag (2004).

[15] S.G. Rajeev, Non Abelian Bosonization without Wess-Zumino terms. 1. New current algebra, Phys. Lett. B 217 (1989) 123 [INSPIRE].

[16] S.G. Rajeev, Nonabelian Bosonization Without Wess-Zumino Terms. 2, Aug. 1988, UR-1088.

[17] K. Sfetsos, Poisson-Lie T duality beyond the classical level and the renormalization group, Phys. Lett. B 432 (1998) 365 [hep-th/9803019] [INSPIRE].

[18] A. Stern, Hamiltonian approach to Poisson Lie T-duality, Phys. Lett. B 450 (1999) 141 [hep-th/9811256] [INSPIRE].

[19] A. Stern, T duality for coset models, Nucl. Phys. B 557 (1999) 459 [hep-th/9903170] [INSPIRE]. 
[20] F. Falceto and K. Gawędzki, Boundary G/G theory and topological Poisson-Lie sigma model, Lett. Math. Phys. 59 (2002) 61 [hep-th/0108206] [INSPIRE].

[21] I. Calvo, F. Falceto and D. Garcia-Alvarez, Topological Poisson sigma models on Poisson lie groups, JHEP 10 (2003) 033 [hep-th/0307178] [INSPIRE].

[22] F. Bonechi and M. Zabzine, Poisson sigma model over group manifolds, J. Geom. Phys. 54 (2005) 173 [hep-th/0311213] [INSPIRE].

[23] K. Sfetsos and K. Siampos, Quantum equivalence in Poisson-Lie T-duality, JHEP 06 (2009) 082 [arXiv: 0904.4248] [INSPIRE].

[24] P. Ševera, On integrability of 2-dimensional $\sigma$-models of Poisson-Lie type, JHEP 11 (2017) 015 [arXiv: 1709.02213] [INSPIRE].

[25] F. Hassler, Poisson-Lie T-duality in Double Field Theory, arXiv:1707.08624 [INSPIRE].

[26] B. Jurčo and J. Vysoky, Poisson-Lie T-duality of string effective actions: A new approach to the dilaton puzzle, J. Geom. Phys. 130 (2018) 1 [arXiv:1708.04079] [InSPIRE].

[27] A. Chatzistavrakidis, L. Jonke, F.S. Khoo and R.J. Szabo, Double Field Theory and Membrane Sigma-Models,, JHEP 07 (2018) 015 [arXiv: 1802.07003] [INSPIRE].

[28] K. Sfetsos, Duality invariant class of two-dimensional field theories, Nucl. Phys. B 561 (1999) 316 [hep-th/9904188] [INSPIRE].

[29] R.A. Reid-Edwards, Bi-Algebras, Generalised Geometry and T-duality, arXiv:1001.2479 [INSPIRE].

[30] N. Hitchin, Generalized Calabi-Yau manifolds, Quart. J. Math. 54 (2003) 281 [math/0209099] [INSPIRE].

[31] N. Hitchin, Lectures on generalized geometry, arXiv:1008.0973 [INSPIRE].

[32] M. Gualtieri, Generalized Complex Geometry, Ph.D. Thesis, Oxford University, U.K. (2004), math/0401221.

[33] C. Hull and B. Zwiebach, Double Field Theory, JHEP 09 (2009) 099 [arXiv:0904.4664] [INSPIRE].

[34] C. Hull and B. Zwiebach, The Gauge algebra of double field theory and Courant brackets, JHEP 09 (2009) 090 [arXiv:0908.1792] [INSPIRE].

[35] O. Hohm, C. Hull and B. Zwiebach, Generalized metric formulation of double field theory, JHEP 08 (2010) 008 [arXiv:1006.4823] [INSPIRE].

[36] O. Hohm, C. Hull and B. Zwiebach, Background independent action for double field theory, JHEP 07 (2010) 016 [arXiv: 1003.5027] [INSPIRE].

[37] G. Aldazabal, D. Marques and C. Núñez, Double Field Theory: A Pedagogical Review, Class. Quant. Grav. 30 (2013) 163001 [arXiv:1305.1907] [INSPIRE].

[38] A.A. Tseytlin, Duality Symmetric Formulation of String World Sheet Dynamics, Phys. Lett. B 242 (1990) 163 [INSPIRE].

[39] A.A. Tseytlin, Duality symmetric closed string theory and interacting chiral scalars, Nucl. Phys. B 350 (1991) 395 [INSPIRE].

[40] C.M. Hull, A geometry for non-geometric string backgrounds, JHEP 10 (2005) 065 [hep-th/0406102] [INSPIRE]. 
[41] C.M. Hull and R.A. Reid-Edwards, Non-geometric backgrounds, doubled geometry and generalised T-duality, JHEP 09 (2009) 014 [arXiv: 0902.4032] [INSPIRE].

[42] D.S. Berman and D.C. Thompson, Duality Symmetric String and M-theory, Phys. Rept. 566 (2014) 1 [arXiv:1306.2643] [InSPIRE].

[43] D.S. Berman, N.B. Copland and D.C. Thompson, Background Field Equations for the Duality Symmetric String, Nucl. Phys. B 791 (2008) 175 [arXiv:0708.2267] [InSPIRE].

[44] K. Lee and J.-H. Park, Covariant action for a string in "doubled yet gauged" spacetime, Nucl. Phys. B 880 (2014) 134 [arXiv: 1307.8377] [INSPIRE].

[45] J.-H. Park, Comments on double field theory and diffeomorphisms, JHEP 06 (2013) 098 [arXiv: 1304.5946] [INSPIRE].

[46] N.B. Copland, A Double $\sigma$-model for Double Field Theory, JHEP 04 (2012) 044

[arXiv:1111.1828] [INSPIRE].

[47] N.B. Copland, Connecting T-duality invariant theories, Nucl. Phys. B 854 (2012) 575 [arXiv: 1106.1888] [INSPIRE].

[48] F. Pezzella, Some Aspects of the T-duality Symmetric String $\sigma$-model, in Proceedings, 14th Marcel Grossmann Meeting on Recent Developments in Theoretical and Experimental General Relativity, Astrophysics and Relativistic Field Theories (MG14) (In 4 Volumes): Rome, Italy, July 12-18, 2015, vol. 4, pp. 4228-4233, 2017, arXiv:1512.08825 [INSPIRE].

[49] F. Pezzella, Two Double String Theory Actions: Non-Covariance vs. Covariance, PoS (CORFU2014) 158 [arXiv: 1503.01709] [INSPIRE].

[50] L. De Angelis, S.J. Gionti, Gabriele, R. Marotta and F. Pezzella, Comparing Double String Theory Actions, JHEP 04 (2014) 171 [arXiv:1312.7367] [INSPIRE].

[51] I. Bandos, Superstring in doubled superspace, Phys. Lett. B 751 (2015) 408 [arXiv: 1507.07779] [INSPIRE].

[52] S. Groot Nibbelink and P. Patalong, A Lorentz invariant doubled world-sheet theory, Phys. Rev. D 87 (2013) 041902 [arXiv:1207.6110] [INSPIRE].

[53] C.-T. Ma and F. Pezzella, Geometric Low-Energy Effective Action in a Doubled Spacetime, Nucl. Phys. B 930 (2018) 135 [arXiv:1706.03365] [INSPIRE].

[54] W. Siegel, Superspace duality in low-energy superstrings, Phys. Rev. D 48 (1993) 2826 [hep-th/9305073] [INSPIRE].

[55] W. Siegel, Two vierbein formalism for string inspired axionic gravity, Phys. Rev. D 47 (1993) 5453 [hep-th/9302036] [inSPIRE].

[56] W. Siegel, Manifest duality in low-energy superstrings, in International Conference on Strings 93 Berkeley, California, May 24-29, 1993, pp. 353-363, hep-th/9308133 [INSPIRE].

[57] W. Siegel, Manifest Lorentz Invariance Sometimes Requires Nonlinearity, Nucl. Phys. B 238 (1984) 307 [INSPIRE].

[58] I. Bakas and D. Lüst, 3-Cocycles, Non-Associative Star-Products and the Magnetic Paradigm of R-Flux String Vacua, JHEP 01 (2014) 171 [arXiv: 1309.3172] [InSPIRE].

[59] V.G. Kupriyanov and R.J. Szabo, Symplectic realization of electric charge in fields of monopole distributions, Phys. Rev. D 98 (2018) 045005 [arXiv:1803.00405] [INSPIRE].

[60] R.J. Szabo, Quantization of Magnetic Poisson Structures, Fortsch. Phys. 67 (2019) 1910022 [arXiv: 1903.02845] [INSPIRE]. 
[61] R. Jackiw, 3-Cocycle in Mathematics and Physics, Phys. Rev. Lett. 54 (1985) 159 [INSPIRE].

[62] J.F. Carinena, J.M. Gracia-Bondia, F. Lizzi, G. Marmo and P. Vitale, Star-product in the presence of a monopole, Phys. Lett. A 374 (2010) 3614 [arXiv:0912.2197] [INSPIRE].

[63] L. Rosa and P. Vitale, On the $\star$-product quantization and the Duflo map in three dimensions, Mod. Phys. Lett. A 27 (2012) 1250207 [arXiv:1209.2941] [INSPIRE].

[64] V.G. Kupriyanov and P. Vitale, Noncommutative $\mathbb{R}^{d}$ via closed star product, JHEP 08 (2015) 024 [arXiv: 1502.06544] [INSPIRE].

[65] J.M. Gracia-Bondia, F. Lizzi, J.C. Varilly and P. Vitale, The Kirillov picture for the Wigner particle, J. Phys. A 51 (2018) 255203 [arXiv:1711.09608] [InSPIRE].

[66] R.J. Szabo, Quantum field theory on noncommutative spaces, Phys. Rept. 378 (2003) 207 [hep-th/0109162] [INSPIRE].

[67] G. Marmo, P. Vitale and A. Zampini, Noncommutative differential calculus for Moyal subalgebras, J. Geom. Phys. 56 (2006) 611 [hep-th/0411223] [INSPIRE].

[68] G. Marmo, P. Vitale and A. Zampini, Derivation based differential calculi for noncommutative algebras deforming a class of three dimensional spaces, J. Geom. Phys. 136 (2019) 104 [arXiv: 1805.06300] [InSPIRE].

[69] P. Martinetti, P. Vitale and J.-C. Wallet, Noncommutative gauge theories on $\mathbb{R}_{\theta}^{2}$ as matrix models, JHEP 09 (2013) 051 [arXiv: 1303.7185] [INSPIRE].

[70] H. Grosse and R. Wulkenhaar, Renormalization of $\phi^{4}$ theory on noncommutative $R^{4}$ in the matrix base,, Commun. Math. Phys. 256 (2005) 305 [hep-th/0401128] [INSPIRE].

[71] R. Gurau, J. Magnen, V. Rivasseau and A. Tanasa, A translation-invariant renormalizable non-commutative scalar model, Commun. Math. Phys. 287 (2009) 275 [arXiv:0802.0791] [INSPIRE].

[72] A. Tanasa and P. Vitale, Curing the UV/IR mixing for field theories with translation-invariant star products, Phys. Rev. D 81 (2010) 065008 [arXiv:0912.0200] [INSPIRE].

[73] M. de Cesare, M. Sakellariadou and P. Vitale, Noncommutative gravity with self-dual variables, Class. Quant. Grav. 35 (2018) 215009 [arXiv: 1806.04666] [INSPIRE].

[74] P. Aschieri and L. Castellani, Noncommutative D $=4$ gravity coupled to fermions, JHEP 06 (2009) 086 [arXiv: 0902.3817] [INSPIRE].

[75] R. Blumenhagen, F. Hassler and D. Lüst, Double Field Theory on Group Manifolds, JHEP 02 (2015) 001 [arXiv: 1410.6374] [INSPIRE].

[76] R. Blumenhagen, P. du Bosque, F. Hassler and D. Lüst, Generalized Metric Formulation of Double Field Theory on Group Manifolds, JHEP 08 (2015) 056 [arXiv:1502.02428] [INSPIRE].

[77] S. Demulder, F. Hassler and D.C. Thompson, Doubled aspects of generalised dualities and integrable deformations, JHEP 02 (2019) 189 [arXiv:1810.11446] [INSPIRE].

[78] H. Mori, S. Sasaki and K. Shiozawa, Doubled Aspects of Vaisman Algebroid and Gauge Symmetry in Double Field Theory, arXiv:1901.04777 [INSPIRE].

[79] V.E. Marotta, F. Pezzella and P. Vitale, Doubling, T-duality and Generalized Geometry: a Simple Model, JHEP 08 (2018) 185 [arXiv: 1804.00744] [INSPIRE]. 
[80] S.G. Rajeev, G. Sparano and P. Vitale, Alternative canonical formalism for the Wess-Zumino-Witten model, Int. J. Mod. Phys. A 9 (1994) 5469 [hep-th/9312178] [INSPIRE].

[81] S.G. Rajeev, A. Stern and P. Vitale, Integrability of the Wess-Zumino-Witten model as a nonultralocal theory, Phys. Lett. B 388 (1996) 769 [hep-th/9602149] [InSPIRE].

[82] G. Marmo and A. Ibort, A new look at completely integrable systems and double Lie groups, Contemp. Math. 219 (1998) 159.

[83] L. Freidel, R.G. Leigh and D. Minic, Born Reciprocity in String Theory and the Nature of Spacetime, Phys. Lett. B 730 (2014) 302 [arXiv:1307.7080] [INSPIRE].

[84] L. Freidel, F.J. Rudolph and D. Svoboda, Generalised Kinematics for Double Field Theory, JHEP 11 (2017) 175 [arXiv:1706.07089] [INSPIRE].

[85] L. Freidel, F.J. Rudolph and D. Svoboda, A Unique Connection for Born Geometry, arXiv: 1806.05992 [INSPIRE].

[86] D. Svoboda, Algebroid Structures on Para-Hermitian Manifolds, J. Math. Phys. 59 (2018) 122302 [arXiv: 1802.08180] [INSPIRE].

[87] V.E. Marotta and R.J. Szabo, Para-Hermitian Geometry, Dualities and Generalized Flux Backgrounds, Fortsch. Phys. 67 (2019) 1800093 [arXiv:1810.03953] [INSPIRE].

[88] C. Klimčík, $\eta$ and $\lambda$ deformations as E-models, Nucl. Phys. B 900 (2015) 259 [arXiv: 1508.05832] [INSPIRE].

[89] G. Marmo, A. Simoni and A. Stern, Poisson lie group symmetries for the isotropic rotator, Int. J. Mod. Phys. A 10 (1995) 99 [hep-th/9310145] [InSPIRE].

[90] A.Yu. Alekseev and A.Z. Malkin, Symplectic structures associated to Lie-Poisson groups, Commun. Math. Phys. 162 (1994) 147 [hep-th/9303038] [INSPIRE].

[91] E.K. Sklyanin, Some algebraic structures connected with the Yang-Baxter equation, Funct. Anal. Appl. 16 (1982) 263 [INSPIRE].

[92] Ya.I. Granovskii, I.M. Lutzenko and A.S. Zhedanov, Mutual integrability, quadratic algebras, and dynamical symmetry, Annals Phys. 217 (1992) 1.

[93] A. Deser and J. Stasheff, Even symplectic supermanifolds and double field theory, Commun. Math. Phys. 339 (2015) 1003 [arXiv:1406.3601] [InSPIRE].

[94] A. Deser and C. Saemann, Extended Riemannian Geometry I: Local Double Field Theory, ITP-UH-22-16, EMPG-16-18, [arXiv: 1611. 02772].

[95] E. Witten, Nonabelian Bosonization in Two-Dimensions, Commun. Math. Phys. 92 (1984) 455 [INSPIRE].

[96] G. Bhattacharya and S. Rajeev, Boson-Fermion Equivalence in a Two-dimensional Anomalous Chiral Model, Nucl. Phys. B 246 (1984) 157 [InSPIRE].

[97] F. Bascone, F. Pezzella and P. Vitale, work in progress.

[98] J.M. Maldacena and H. Ooguri, Strings in $A d S_{3}$ and $\mathrm{SL}(2, \mathbb{R})$ WZW model. Part I: The spectrum, J. Math. Phys. 42 (2001) 2929 [hep-th/0001053] [inSPIRE].

[99] J.M. Maldacena, H. Ooguri and J. Son, Strings in $A d S_{3}$ and the $\mathrm{SL}(2, \mathbb{R}) W Z W$ model. Part 2. Euclidean black hole, J. Math. Phys. 42 (2001) 2961 [hep-th/0005183] [INSPIRE].

[100] J.M. Maldacena and H. Ooguri, Strings in $A d S_{3}$ and the $\mathrm{SL}(2, \mathbb{R})$ WZW model. Part 3. Correlation functions, Phys. Rev. D 65 (2002) 106006 [hep-th/0111180] [INSPIRE]. 